\title{
Tet1 Isoforms Differentially Regulate Gene Expression, Synaptic Transmission, and Memory in the Mammalian Brain
}

\author{
${ }^{\circledR}$ C.B. Greer, ${ }^{1}$ J. Wright, ${ }^{1}$ J.D. Weiss, ${ }^{1}{ }^{\circledR}$ R.M. Lazarenko, ${ }^{1}$ S.P. Moran, ${ }^{1}{ }^{\circledR}$ J. Zhu, ${ }^{1}$ K.S. Chronister, ${ }^{1}{ }^{1}$ A.Y. Jin, ${ }^{1}$ \\ ${ }^{\circ}$ A.J. Kennedy, ${ }^{2}{ }^{\circledR}$ J.D. Sweatt, ${ }^{1}$ and ${ }^{\circ}$ G.A. Kaas ${ }^{1}$ \\ ${ }^{1}$ Department of Pharmacology, Vanderbilt University, Nashville, Tennessee 37232, and ${ }^{2}$ Department of Chemistry, Bates College, Lewiston, Maine \\ 04240
}

The dynamic regulation of DNA methylation in postmitotic neurons is necessary for memory formation and other adaptive behaviors. Ten-eleven translocation 1 (TET1) plays a part in these processes by oxidizing 5-methylcytosine $(5 \mathrm{mC})$ to 5-hydroxymethylcytosine $(5 \mathrm{hmC})$, thereby initiating active DNA demethylation. However, attempts to pinpoint its exact role in the nervous system have been hindered by contradictory findings, perhaps due in part, to a recent discovery that two isoforms of the Tet1 gene are differentially expressed from early development into adulthood. Here, we demonstrate that both the shorter transcript $\left(\mathrm{Tet}_{1}{ }^{S}\right)$ encoding an N-terminally truncated TET1 protein and a full-length Tet1 $\left(\right.$ Tet $\left.1^{F L}\right)$ transcript encoding canonical TET1 are co-expressed in the adult mouse brain. We show that $\mathrm{Tetl}^{S}$ is the predominantly expressed isoform and is highly enriched in neurons, whereas $T e t 1^{F L}$ is generally expressed at lower levels and more abundant in glia, suggesting their roles are at least partially cell type-specific. Using viral-mediated, isoform and neuron-specific molecular tools, we find that the individual repression of each transcript leads to the dysregulation of unique gene ensembles and contrasting changes in basal synaptic transmission. In addition, $\mathrm{Tet}^{S}$ repression enhances, while $\mathrm{Tetl}^{\mathrm{FL}}$ impairs, hippocampal-dependent memory in male mice. Together, our findings demonstrate that each Tet1 isoform serves a distinct role in the mammalian brain.

Key words: epigenetics; gene expression; memory; neurons; synaptic transmission; TET1

Significance Statement

In the brain, activity-dependent changes in gene expression are required for the formation of long-term memories. DNA methylation plays an essential role in orchestrating these learning-induced transcriptional programs by influencing chromatin accessibility and transcription factor binding. Once thought of as a stable epigenetic mark, DNA methylation is now known to be impermanent and dynamically regulated, driving neuroplasticity in the brain. We found that Tet1, a member of the teneleven translocation (TET) family of enzymes that mediates removal of DNA methyl marks, is expressed as two separate isoforms in the adult mouse brain and that each differentially regulates gene expression, synaptic transmission and memory formation. Together, our findings demonstrate that each Tet1 isoform serves a distinct role in the CNS.

Received July 14, 2020; revised Sep. 28, 2020; accepted Nov. 2, 2020.

Author contributions: C.B.G., J.D.S., and G.A.K. designed research; C.B.G., J.W., J.D.W., R.M.L., S.P.M., J.Z., K.S.C., and G.A.K. performed research; C.B.G., S.P.M., A.Y.J., and G.A.K. analyzed data; C.B.G., S.P.M., A.J.K., J.D.S., and G.A.K. edited the paper; G.A.K. wrote the paper.

This work was supported by National Institutes of Health Grants MH057014 and MH107254 (to J.D.S.), P20-GM103423 (to A.J.K.), T32-MH065215 (to C.B.G.), and U54-HD083211 (to the Vanderbilt Mouse Neurobehavior Lab). We thank Hehuang Xie, Roger J. Colbran, Danny G. Winder, and Colleen Niswender for helpful comments and discussions during the preparation of this manuscript. We also thank the Murine Neurobehavioral Core lab at the Vanderbilt University Medical Center for their guidance and use of equipment.

The authors declare no competing financial interests.

Correspondence should be addressed to G. A. Kaas at garrett.kaas@vanderbilt.edu.

https://doi.org/10.1523/JNEUROSCI.1821-20.2020

Copyright $\odot 2021$ Greer et al.

This is an open-access article distributed under the terms of the Creative Commons Attribution 4.0 International license, which permits unrestricted use, distribution and reproduction in any medium provided that the original work is properly attributed.

\section{Introduction}

DNA methylation is an essential regulator of gene expression in the brain, and is required for learning and memory formation (Jarome and Lubin, 2014). Based on its role during development, DNA methylation was initially thought to function as a stable epigenetic mark in postmitotic brain cells, but it is now known to be dynamically regulated, in response to neuronal stimulation, learning, and experience (Martinowich et al., 2003; Miller and Sweatt, 2007; Saunderson et al., 2016). DNA methylation levels are controlled by the antagonistic actions of DNA methyltransferases (DNMTs), which methylate the fifth carbon of cytosine bases [5-methylcytosine (5mC); Okano et al., 1999; Hermann et al., 2004], and the ten-eleven translocation (TET) enzymes, which oxidize $5 \mathrm{mCs}$ to 5 -hydroxymethylcytosine $(5 \mathrm{hmC})$ and 
initiate active DNA demethylation (Tahiliani et al., 2009; Guo et al., 2011). TET enzymes are critical for brain function and mutations or changes in the expression of Tet genes are associated with, or the cause of, cognitive deficits in humans (Dong et al., 2015; Cochran et al., 2020; Beck et al., 2020). Thus, the study of TET-mediated mechanisms in the brain may provide novel insights into the pathophysiology of neurologic disease.

All three Tet genes (Tet1-3) are expressed in the mammalian brain and studies suggest they generally serve non-redundant functions. Tet 3 is the highest expressed and is transcriptionally upregulated by neuronal stimulation (Widagdo et al., 2014). Knock-down (KD) of Tet3 alters synaptic transmission, and conditional knock-out $(\mathrm{KO})$ of the gene impairs spatial memory, indicating that its necessary for cognition ( $\mathrm{Yu}$ et al., 2015; Antunes et al., 2020). Tet2 is also abundantly expressed in the brain and its disruption is associated with enhanced spatial memory, suggesting it may function as a negative regulator of neuroplasticity (Zengeler et al., 2019). Tet1, despite much lower expression, has been the most studied Tet family member in the nervous system, implicated in the regulation of activity-dependent gene expression, synaptic transmission, and cognition (Alaghband et al., 2016). However, attempts to define its exact role, particularly in the context of learning and memory, have been hampered by contradictory findings. For instance, depending on the study, loss of Tet1 in KO mice has been reported to either impair, enhance, or have no effect at all on memory (Rudenko et al., 2013; Zhang et al., 2013; Kumar et al., 2015; Towers et al., 2018). Likewise, overexpression of Tet 1 has been shown to enhance memory, while expression of its catalytic domain does the opposite (Kaas et al., 2013; Kwon et al., 2018). One potential explanation for these inconsistences comes from a recent report that the Tet1 gene undergoes an isoform switch from the full-length, canonical transcript (hereafter $T e t 1^{F L}$ ) in embryonic stem cells to a shorter, truncated variant (hereafter $T e t 1^{S}$ ) exclusively expressed to somatic tissues (Zhang et al., 2016). In addition, evidence suggests that in some tissues both transcripts might be co-expressed (Good et al., 2017; Yosefzon et al., 2017). Whether this is the case in the adult brain, and if so, what functions these Tet1 isoforms might serve, has not been explored.

Here, we report that both Tet 1 isoforms are expressed in the brain. The Tet $1^{S}$ isoform is highly enriched in neurons and its expression is regulated in an activity-dependent manner. In contrast, $T e t 1^{F L}$ is transcribed at low basal levels in neurons, yet is much more abundant in glia, suggesting the functions of each isoform may be at least partially cell-type specific. Using newly-developed molecular tools, we found that the transcriptional repression of each individual isoform results in distinct changes in neuronal gene expression, basal synaptic transmission and memory formation, demonstrating that $T e t 1^{F L}$ and $T e t 1^{S}$ serve important, non-redundant functions in the nervous system.

\section{Materials and Methods \\ Animals}

Experiments were performed using two- to three-month-old C57BL/6J male mice originally purchased from The Jackson Laboratory. All mice were group housed, kept under 12:12 light/dark cycles, with food and water available ad libitum. For stereotaxic surgeries and behavioral assays, four-week-old male littermates were purchased, housed in sets of three to five animals and aged out to 10 weeks before experimentation in an effort to minimize fighting. All procedures and behavioral assays were approved by the Vanderbilt Animal Care and Use Committee.

\section{Chromatin immunoprecipitation (ChIP)}

Tissue samples were cut into $\sim 1-\mathrm{mm}$ pieces using a razor and incubated in a $1 \times$ PBS solution containing $1 \%$ formaldehyde and proteinase inhibitors for $10 \mathrm{~min}$ at $37^{\circ} \mathrm{C}$, followed by the addition of glycine (final concentration $125 \mathrm{~mm}$ ) to quench the reaction. Samples were washed $6 \times$ with ice-cold PBS and then homogenized in lysis buffer (50 mM Tris, $\mathrm{pH}$ 8.1, $10 \mathrm{~mm}$ EDTA, and 1\% SDS) using a pestle. Chromatin was sheared using a Bioruptor Pico set to three cycles of $30 \mathrm{~s}$ on, $30 \mathrm{~s}$ off. Samples were then processed using the Magna ChIP G Tissue kit (EMD Millipore) according to manufacturer instructions. Briefly, samples were precleared using $25 \mu \mathrm{l}$ of Protein G beads, then placed on a rotator and incubated overnight at $4^{\circ} \mathrm{C}$ with $25 \mu \mathrm{l}$ of Protein G beads and $4 \mu \mathrm{l}$ ( $1 \mathrm{mg} / \mathrm{ml}$ ) of anti-RNA polymerase II antibody (102660; Active Motif). Immune complexes were sequentially washed according to kit instructions. To reverse cross-links, samples were incubated at $65^{\circ} \mathrm{C}$ for $2 \mathrm{~h}$ in the presence of SDS and Proteinase K, then at $95^{\circ} \mathrm{C}$ for $10 \mathrm{~min}$. Enriched DNA samples were purified with a QIAGEN PCR clean up kit. Purified DNA was then stored at $-20^{\circ} \mathrm{C}$ or used immediately for quantitative real-time PCR (qRT-PCR). Fold enrichment for each primer set was normalized to input and reported relative to a negative control gene desert region (mouse Igxla locus; QIAGEN). Primers: Tet $^{F L}$ promoter $\mathrm{F}$ $5^{\prime}$-gcactctgcaactggtttg-3', R $5^{\prime}$-gtagaagaggcaggtagaggta- $3^{\prime} ;$ Tet $^{S}$ promoter F $5^{\prime}$-ctgctttgaaacaccatgataa-3', R 5'-tagccatcttgcctgctt-3'.

\section{$5^{\prime}$ rapid amplification of $c D N A$ ends (RACE)}

Total RNA was extracted from adult mouse hippocampal tissue using an RNeasy Plus kit (QIAGEN). Amplification of $5^{\prime} \mathrm{cDNA}$ ends was performed using the GeneRacer kit (Life Technologies) in accordance with manufacturer instructions. Briefly, 3- $\mu$ g total RNA was dephosphorylated, decapped, and then ligated to a GeneRacer 5' RNA oligo. Between each step, samples were purified by phenol:chloroform extraction and precipitated with ethanol and glycogen. cDNA was then synthesized from the ligated RNA using Superscript III reverse transcriptase (Invitrogen) and random primers. cDNA samples were amplified for two rounds by nested PCR using the Platinum PCR Supermix High Fidelity (Invitrogen) kit and $5^{\prime}$ GeneRacer forward and reverse Tet1-isoform specific primers (Tet ${ }^{F L}$ 5' RACE outside, 5' -ttgggtgtgactactgggcgctgggaga- $3^{\prime} ;$ Tet1 ${ }^{F L}$ RACE nested, $5^{\prime}$ ggcgctgggagagtcgccagctaaga- $3^{\prime}$; Tet $1^{S} 5^{\prime}$ RACE outside, $5^{\prime}$-agccaggcttctggaagagcagggtgt- $3^{\prime}$; Tet $1^{S}$ RACE nested, $5^{\prime}$-cccggaggtggtgacactcatggcatcctt-3'). Purified PCR products were then cloned into pCR-Blunt II-TOPO vectors (Invitrogen) and subjected to sanger sequencing (GenHunter Corp.).

\section{$q R T-P C R$}

Total RNA was extracted from samples using an RNeasy Plus Mini kit (QIAGEN) and eluted in 30-50 $\mu$ l of RNase free water. Sample concentration and purity were analyzed using a NanoDrop One Microvolume UV-Vis Spectrophotometer (Thermo Scientific) and cDNA synthesis was conducted in $20-\mu \mathrm{l}$ reactions using the iScript cDNA Synthesis kit (Bio-Rad), or $10-\mu$ l reaction using SuperScript VILO master mix (Invitrogen) according to manufacturer instructions. All cDNA reactions were diluted 1:5 with RNase-free water to reduce the influence of any PCR inhibitors. qRT-PCR was performed on an CFX96 RT-PCR detection system in $10-\mu$ l reactions containing SsoAdvanced Universal SYBR Green Supermix and 200-300 $\mu \mathrm{m}$ primer and $1 \mu \mathrm{l}$ of cDNA. All qRT-PCR primers were either designed using Primer Quest (Integrated DNA Technologies) to span exon-exon junctions or were acquired directly as predesigned PrimeTime qPCR Primer Assays (Integrated DNA Technologies). Relative fold quantification of gene expression between samples was calculated using the comparative $\mathrm{C}_{\mathrm{t}}$ method (Livak and Schmittgen, 2001)and normalized to the geometric mean of three reference genes Hypoxanthine phosphoribosyltransferase (Hprt), Glyceraldehyde-3-Phosphate Dehydrogenase (Gapdh), and Glucuronidase Beta (Gusb). IDT PrimeTime qPCR Probe Assays: Hprt, Mm.PT.39a.22214828; Gapdh, Mm.PT.39a.1. Primers: Gusb, F 5'cagactcagttgttgtcacct- $3^{\prime}, \quad \mathrm{R} \quad 5^{\prime}$-tcaacttcaggttcccagtg-3'; Tet ${ }^{F L} \mathrm{~F}$ $5^{\prime}$-ctccctggtcatgtacctcta-3', R $5^{\prime}$-gtaagtaaagatgcaaggatgcg-3'; Tet ${ }^{S} \mathrm{~F}$ $5^{\prime}$-cctccatctttatttatgcaag- $3^{\prime}, \mathrm{R} 5^{\prime}$-ggtttgttgttaaagtctgtct- $3^{\prime}$; Neuronal PAS domain protein 4 (Npas4) F 5'-cacgtcttgatgacaatatgcc-3', R 5'ccaagttcaagacagcttcca-3'; Activity regulated cytoskeleton associated 
protein $(A r c)$ F $55^{\prime}$-acgatctggcttcctcattctgct-3', R $5^{\prime}$-aggttccctcagcatctctgcttt-3'; Early growth response 1 (Egr1) F 5'-agcgccttcaatcctcaag-3', R 5'-tttggctgggataactcgtc- $3^{\prime}$.

\section{Mouse primary neuron, glia and neuroblastoma 2 a cultures}

Mouse hippocampi from C57BL/6J P0 pups were dissected in ice-cold HBSS (Invitrogen) and digested with papain (Worthington) for $25 \mathrm{~min}$ at $37^{\circ} \mathrm{C}$. Samples were then washed $2 \times$ in HBSS and dissociated by pipetting up and down 15-20 times through a P1000 pipette in growth media [Neurobasal media supplemented with $5 \%$ fetal bovine serum (FBS), $500 \mathrm{~nm}$ L-glutamine, $1 \times$ B27 (Invitrogen), and PenicillinStreptomycin (Pen-Strep)]. The cell suspension was passed through a $100 \mu \mathrm{m}$ filter and centrifuged for $5 \mathrm{~min}$ at $500 \times \mathrm{g}$. Cell pellets were resuspended in growth media and seeded on poly-D-lysine coated (Sigma) 12-well plates (Corning) at $\sim 250-300 \times 10^{3}$ cells per well or on 24 -well plates at $100 \times 10^{3}$ cells per well; $24 \mathrm{~h}$ later, media was replaced with maintenance media (Neurobasal media supplemented with $500 \mathrm{~nm}$ L-glutamine, $1 \times$ B27) without Pen-Strep. At Days in vitro (DIV) 3-4, $1 \mu \mathrm{M}$ 5-fluorodeoxyuridine (FdU) was added to the media for $24 \mathrm{~h}$, then removed, to inhibit mitotic cell growth (Hui et al., 2016). Primary mixed glial cultures were prepared identically to neurons, except they were plated, then maintained, using media that consisted of $1 \times$ DMEM (+4.5 g/l D-glucose and L-glutamine, Invitrogen), PenStrep and 10\% FBS. DIV10-DIV14 neuronal and glial cultures were used for qRT-PCR experiments. GSK126 or GSK343 were dissolved in Dimethyl sulfoxide (DMSO), and applied to cells for $72 \mathrm{~h}$. Stimulation of neurons was conducted by adding $25 \mu \mathrm{l}$ of maintenance media alone (vehicle), or media containing $\mathrm{KCl}$, bicuculline, and NMDA/glycine (gly; final concentrations $25 \mathrm{mM}, 50 \mu \mathrm{M}, 10 \mu \mathrm{M} / 2 \mu \mathrm{M}$, respectively). Drugs were administered to each well and incubated for 1,2 , or $4 \mathrm{~h}$. Mouse neuroblastoma 2a (N2a) cells were purchased from ATCC, and cultured using the same media as glial cells. For transfection experiments, N2a cells were seeded in 24 -well plates at a density of $200 \times 10^{3}$ per well and transfected using GenJet Reagent (II) in accordance with manufacturer instructions.

\section{Transcription activator like effector (TALE) vector construction and analysis}

All constructs used in this study were generated using Gibson Assembly methodology as previously described (Gibson et al., 2009). Briefly, primers were designed using NEBuilder Assembly Tool (v1.12.18) to generate overlapping (20-25 bp) PCR fragments amplified using Q5 High-Fidelity DNA Polymerase (NEB). Modified TALE constructs were created using PCR fragments amplified from pAAV-CW3SL-EGFP (Addgene \#61463), a gift from Bong-Kiun Kaang (Choi et al., 2014), and constructs contained in the TALE Toolbox (Addgene kit \#1000000019), a gift from Feng Zhang. TALE DNA targeting sequences were assembled into TALE construct backbones using Golden Gate Assembly Cloning, as previously described (Sanjana et al., 2012). TALE sequences targeting the Tet $1^{F L}$ (5'-TGCCCCAGCTACACTCCT-3', sense) and Tet ${ }^{S^{S}}{ }^{\prime} 5^{\prime}$ TCGCAGCCTAGCACTATC-3', antisense) promoter regions were designed using TAL Effector Nucleotide Targeter 2.0 software (Doyle et al., 2012). Potential off-target genes for each sequence were identified using a UNIX shell script relying on bowtie (Langmead et al., 2009) with option -a to report all alignments and $-\mathrm{v}$ three to find sites with up to three mismatches in the mouse genome (mm10). The output was converted with samtools (Li et al., 2009), and homer annotatePeaks.pl (Heinz et al., 2010) was used to annotate the location of mismatched sequences relative to annotated genes and to determine the proximity to the promoter. Genes previously identified as TET1 targets in ChIP sequencing (ChIP-seq) datasets were excluded (Lachmann et al., 2010).

\section{Immunostaining}

For immunocytochemistry, cells were plated on glass cover slips coated with poly-D-lysine. Cells were washed $2 \times$ with ice cold $1 \times$ PBS, followed by fixation with fresh $4 \%$ paraformaldehyde for $15 \mathrm{~min}$ at room temperature (RT), blocked for $1 \mathrm{~h}$ at RT (10\% goat serum and $0.3 \%$ Triton X100 in $1 \times$ PBS), and incubated with primary antibodies (anti-HA, ab18181; anti-GFP, ab13970; anti-NeuN, ab104224; Abcam) at a concentration of 1:1000 at RT for $2 \mathrm{~h}$ or overnight at $4^{\circ} \mathrm{C}$ in 1:3 diluted blocking buffer. Slides were then washed and incubated with the appropriate Alexa Flour secondary antibodies (Abcam) at a concentration of 1:1000 for $1 \mathrm{~h}$ at RT. Slides were mounted using ProLong Gold Antifade Mountant with DAPI (Invitrogen) and images were acquired using a IX73 microscope (Olympus) and cellSens standard software.

\section{$A A V$ generation and viral injections}

High titers $\left(>10^{13}\right.$ genome copies $\left./ \mathrm{ml}\right)$ of AAV1 viral particles containing the Tet1 isoform TALE constructs were packaged by Applied Biological Materials (ABM). For primary hippocampal neuron experiments in 12-well or 24-well plates, we added $1 \mu \mathrm{l}$ of AAVs diluted 1:10. For in vivo experiments involving stereotaxic surgeries, AAVs were injected bilaterally into the dorsal hippocampus (dHPC) of 10-week-old mice using the following stereotaxic coordinates: $-2.0 \mathrm{~mm} \mathrm{AP}, \pm 1.5$ $\mathrm{mm} \mathrm{ML}$, and $-1.6 \mathrm{~mm} \mathrm{DV}$ from bregma. A total of $1.5 \mu \mathrm{l}$ of viral solution was injected per hemisphere. Injections were performed using a 10$\mathrm{ml}$ Hamilton Gastight syringe controlled by a Pump 11 Elite Nanomite Programmable Syringe Pump (Harvard Apparatus). Injections proceeded at a speed of $150 \mathrm{nl} \mathrm{min}{ }^{-1}$ through a 32-gauge needle. The injection needle was left in place an additional $5 \mathrm{~min}$. Behavior and gene expression experiments involving AAV delivery in vivo, were either begun or performed 14 days after surgery, respectively.

\section{Behavior}

All behavioral assays were conducted in the Vanderbilt Mouse Neurobehavioral Core (https://lab.vanderbilt.edu/mouse-core/) by blinded experimenters.

Elevated zero maze (EZM). Mice were placed on the open section of the maze (White 2325-0229, San Diego Instruments) and allowed to explore freely for $5 \mathrm{~min}$. Video recording and tracking were performed using ANY-maze video tracking software (Stoelting Co).

Open field. Mice were placed in the center of a large Plexiglas box $(43 \times 42 \times 30 \mathrm{~cm})$, and locomotor activity was measured for $30 \mathrm{~min}$ (Med Associates). Data are presented as total distance traveled in centimeters.

Contextual fear conditioning $(C x F C)$. Fear conditioned mice used for behavioral analysis were trained in a novel context (catalog \#MEDVFC2-SCT-M, Med Associates Inc.) using a 3.5-min training protocol consisting of a 3-min habituation period, followed by a single foot shock $(0.5 \mathrm{~mA}, 2 \mathrm{~s})$. Mice were removed from the chamber $30 \mathrm{~s}$ later. To assess long-term memory formation, mice were placed back in the same context for $5 \mathrm{~min}$ in the absence of the unconditioned stimulus. Percent freezing was calculated automatically using Video Freeze version 2.1.0 (Med Associates Inc.). Fear conditioned animals used for gene expression analysis were trained using a protocol consisting of three contextshock pairings as described above $(0.75 \mathrm{~mA}, 2$ s) every $2 \mathrm{~min}$, and removed from the apparatus after $7 \mathrm{~min}$. At the conclusion of behavioral testing, hippocampi were removed from all animals and AAV1mediated enhanced green fluorescent protein (EGFP) expression was examined using an IX73 microscope (Olympus). Because of the reported role of TET1 in neurogenesis (Zhang et al., 2013), any animals displaying EGFP expression in the dentate gyrus were excluded from our study.

\section{RNA-seq and ChIP-seq}

For RNA-seq, total RNA was extracted from AAV1-transduced primary hippocampal neurons (DIV12-DIV14) using an AllPrep DNA/RNA/ Protein Mini kit (QIAGEN). Total RNA was poly A selected and sequenced (Hudson Alpha GSL) on the Illumina platform (HiSeq v.4, paired end, $50 \mathrm{bp}, 50$ million reads). Reads were passed through a quality filter with trimmomatic (Bolger et al., 2014) using recommended settings for paired end libraries, and adaptor sequences matching TruSeq3_PE were trimmed. Surviving reads were aligned to the mm10 genome with hisat2 (Kim et al., 2015). Stringtie (Pertea et al., 2015) was used to incorporate any novel transcripts from these sequencing libraries into the mm10 Refseq annotation of known transcripts, and featurecounts (Liao et al., 2014) attributed reads to the resulting custom annotation. 
EdgeR (Robinson et al., 2010; McCarthy et al., 2012) was used for determining fold change and false discovery rates (FDRs) for each gene with sufficient read depth. Statistics in EdgeR were determined with genewise negative binomial generalized linear models with quasi-likelihood tests (glmQLFit function). H3K4me3 ChIP-seq from CA1 neurons was downloaded from (Halder et al., 2016). Alignment, peak calling, and file visualization were conducted as described previously (Collins et al., 2019). RNA-seq datasets generated in this study have been deposited in Gene Expression Omnibus (GEO) with the accession number GSE140174.

\section{Electrophysiology}

Whole-cell voltage clamp recordings were performed on neurons from 14 to 21 DIV mouse hippocampal cultures using a Multi-Clamp 700B amplifier. Signals were digitized through a Digidata $1440 \mathrm{~A}$ at $20 \mathrm{kHz}$, filtered at $1.8 \mathrm{kHz}$, and analyzed offline with Clampfit 10.7 software (Molecular Devices). Cells were held at $-60 \mathrm{mV}$. Patch pipettes were pulled from borosilicate glass capillaries with resistances ranging from 3 to $6 \mathrm{M} \Omega$ when filled with pipette solution, containing the following: $120 \mathrm{mM}$ cesium methanesulfonate, $5 \mathrm{mM} \mathrm{CsCl}, 1 \mathrm{mM} \mathrm{MgCl}, 1 \mathrm{mM}$

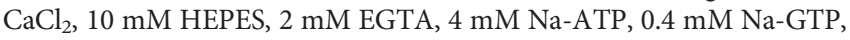
$10 \mathrm{mM}$ phosphocreatine, $3 \mathrm{mM} \mathrm{Na}$-ascorbate, and $5 \mathrm{mM}$ glucose, $\mathrm{pH}$ 7.2. The bath solution (Tyrode's saline) contained the following: $150 \mathrm{mM}$ $\mathrm{NaCl}, 4 \mathrm{mM} \mathrm{KCl}, 2 \mathrm{mM} \mathrm{MgCl}, 2 \mathrm{mM} \mathrm{CaCl}_{2}, 10 \mathrm{mM}$ HEPES, and $10 \mathrm{mM}$ glucose, $\mathrm{pH}$ 7.35. For the recordings of miniature excitatory postsynaptic currents (mEPSCs), bath solution was supplied with $1 \mu \mathrm{M}$ tetrodotoxin (TTX, Hello Bio). mEPSC events were collected from the recorded traces using a template-based approach. Templates were generated from traces recorded under control conditions. 30 randomly selected events from individual recordings were used for analysis.

\section{Experimental design and statistical analyses}

Statistical analysis and graphing of non-genomic data were performed using GraphPad prism 7.04. For two groups, statistical significance was determined using the Student's $t$ test. For three or more groups, statistical significance was determined using one-way or two-way analysis of variance (ANOVA), followed by Dunnett's multiple comparisons tests post hoc. Statistical significance of cumulative distributions was determined using the Kolmogorov-Smirnov test.

\section{Results}

Tet1 is expressed as two distinct transcripts in the adult brain In order to establish whether the Tet 1 gene is expressed as more than one transcript in the adult brain, we first examined the Tet 1 5 ' coding region for promoter-associated histone marks using published ChIP-seq datasets derived from adult NeuN+ hippocampal neurons (Halder et al., 2016). We found two regions within the Tet1 gene locus that were enriched with $\mathrm{H} 3$ lysine four tri-methylation (H3K4me3), H3 lysine 27 acetylation (H3K27ac), and $\mathrm{H} 3$ lysine 9 acetylation (H3K9ac); marks typically associated with transcriptionally active promoters (Liang et al., 2004; Gates et al., 2017; Sato et al., 2019). The distal site, termed promoter 1, was located upstream of Tet 1 Refseq exon 1 and only mildly enriched for the three epigenetic modifications, whereas the second region, termed promoter 2, was located in an intronic region just upstream of Tet1 Refseq exon 2 and was characterized by much stronger active histone peaks (Fig. 1A). To test whether like other active promoters, RNA polymerase II (RNAP2) was enriched at these sites, we conducted ChIP on hippocampal chromatin using primers targeted to each region. At both sites, we observed significant RNAP2 enrichment compared with a negative control region (fold change: $F_{(2,15)}=5.2, p=$ 0.0198; one-way ANOVA; negative control, $1 \pm 0.16$ vs site 1 , $2.3 \pm 0.5, p=0.029$, negative control, $1 \pm 0.16$ vs site $2,2.4 \pm 0.3$, $p=0.023, n=6$ for all groups; Dunnett's post hoc test) indicating that each promoter was likely transcriptionally active (Fig. $1 B$ ).
Next, we used hippocampal RNA and 5' RACE to examine whether the transcriptional start sites (TSSs) of any Tet 1 transcripts aligned to either of the predicted promoters. Our analysis identified two separate TSSs that corresponded to each promoter. As is common for many genes, their precise start sites varied by several nucleotides (Giardina and Lis, 1993; Leenen et al., 2016; Fig. 1C). The transcript starting at promoter 1, termed Tet1 full length $\left(\right.$ Tet $\left.^{F L}\right)$, encodes the full length canonical TET1 enzyme translated from a start codon (Kozak sequencegccATGt) located in exon 2 (Refseq Tet1 exon 1). While the transcript arising from intronic promoter 2, termed Tet1 short $\left(\operatorname{Tet} 1^{S}\right)$, encodes for a truncated enzyme lacking a large portion of the TET ${ }^{\mathrm{FL}} \mathrm{N}$ terminus, including the CXXC non-methylated CpG binding domain (Fig. 1D). TET ${ }^{\mathrm{S}}$ is translated from a start codon (Kozak sequence-tccATGg) located in the third exon of the Refseq annotated Tet1 transcript.

In order to examine where, and to what extent, $\operatorname{Tet}^{F L}$ and Tet ${ }^{S}$ were expressed in the brain, we designed isoform-specific primers (Fig. $1 D$ ) to perform qRT-PCR using cDNA libraries generated from the cerebellum, cortex, hippocampus, and striatum. We detected both Tet 1 transcripts in all four brain regions, with the mRNA levels of Tet $1^{S} \sim 10$-fold higher than Tet $1^{F L}$ across all samples (Fig. $1 E$ ), indicating that it is the predominant Tet 1 transcript expressed in the brain. Notably, the levels of both transcripts were an order of magnitude higher in the cerebellum than any of other brain regions examined (Tet1 isoform fold changes Cer: $S, 0.23 \pm 0.049$ vs Cer: $F L, 0.025 \pm 0.011, t_{(4)}=4.2$, $p=0.014$; Ctx: $S, 0.016 \pm 0.0026$ vs Ctx: $F L, 0.0021 \pm 0.0015, t_{(4)}=$ 4.5, $p=0.011$; Hpc: $S, 0.02 \pm 0.0018$ vs Hpc: $F L, 0.0013 \pm 0.0002$, $t_{(4)}=10, p=0.0005$; Str: $S, 0.022 \pm 0.00,057$ vs Str: FL, $0.0013 \pm$ $0.00,049, t_{(4)}=28, p<0.0001, n=3$ all groups; unpaired two-tailed $t$ test). In addition, we measured Tet $1^{F L}$ and Tet $1^{S}$ mRNA levels in the adult heart, kidney, liver, muscle, and spleen. Both transcripts were present, and expressed at ratios comparable to those in the brain, suggesting this Tet 1 expression pattern is a general feature of most somatic tissues (data not shown). Taken together, our results demonstrate that two transcripts, encoding distinct protein isoforms, are actively generated from the Tet1 gene in the adult mammalian brain and that the novel, truncated $\operatorname{Tet}^{S}$ is the predominant transcript.

\section{Tet1 isoform transcript usage and regulation significantly differ between neurons and glia}

Because our initial experiments were conducted using heterogeneous brain tissue, we next compared the expression levels of Tet $1^{F L}$ and $T e t 1^{S}$ in postnatal hippocampal neurons and glia using qRT-PCR. Tet $1^{S}$ was expressed at $\sim 3$-fold higher levels in neurons than in glia ( $\operatorname{Tet}^{S}$ fold change: glia, $0.34 \pm 0.017$ vs neurons, $1 \pm 0.14, t_{(10)}=5, p=0.0005, n=6$ all groups; unpaired two-tailed $t$ test), whereas Tet $1^{F L}$ transcripts were $\sim 15$-fold more abundant in glia than in neurons $\left(\right.$ Tet $^{F L}$ fold change: glia, $15 \pm 0.74$ vs neurons, $1.2 \pm 0.32, t_{(10)}=17, p<0.0001, n=6$ all groups; unpaired two-tailed $t$ test; Fig. $2 A$ ). To explore these differences further, we compared the chromatin status of each Tet 1 isoform promoter in non-neuronal (NeuN-) cells to that of neurons using previously published ChIP-seq datasets from adult hippocampal tissue (Halder et al., 2016). Similar to neurons, we found in NeuN- cells that both Tet1 isoform promoters were coenriched for $\mathrm{H} 3 \mathrm{~K} 4 \mathrm{me} 3, \mathrm{H} 3 \mathrm{~K} 9 \mathrm{ac}$, and $\mathrm{H} 3 \mathrm{~K} 27 \mathrm{ac}$. However, in these cells, the most enriched $\mathrm{H} 3 \mathrm{~K} 4 \mathrm{me} 3$ peak was located at the Tet $1^{F L}$ promoter (Fig. $2 B$ ), while in neurons we observed the strongest enrichment at the $\mathrm{Tet}^{S}$ promoter (Fig. $1 A$ ). In addition, we found that in neurons, the Tet $1^{F L}$ promoter was also 
A

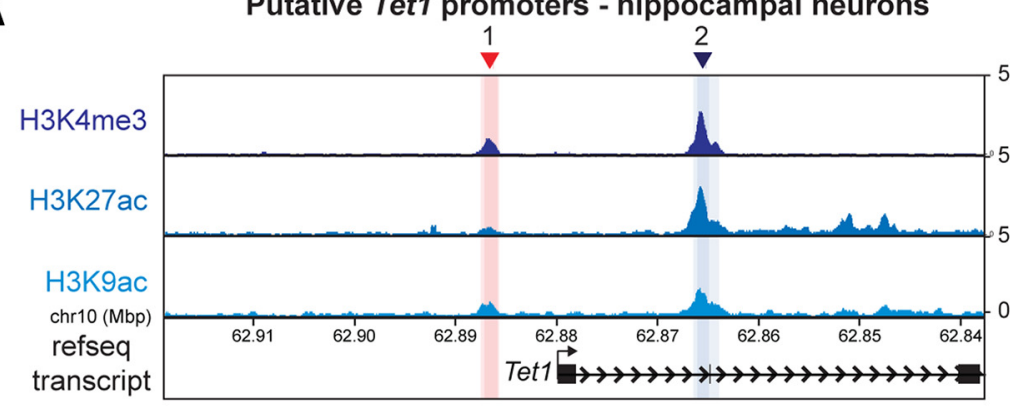

B

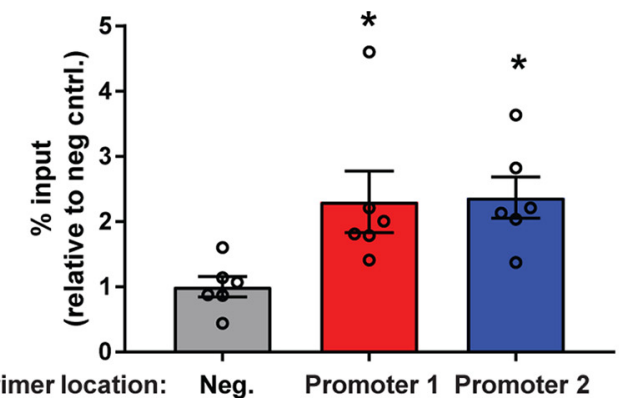

C

5' RACE - hippocampus

TSS sequence within promoter 1 (mm10 chr10:62887172-62887427)

$\Gamma_{\text {GCTACACT }}^{1} \stackrel{5}{\longrightarrow}$

GCTACACTCCTAGAGGTCTGTACTGGGGAGACACCGCTGTCTGGGGGGGGGCGAG GGCTGACCTGGCTGGGAGTACCCGCGCACTCCGCTCGTGGAACACTGCACTCTGC AACTGGTTTGTACAATCCCGGGTCTGTATTCGCCCCTGGACGTCCTCTCCCTCCCTGGTCATGTACCTCTACCTGCCTCTTCTACGGGAACATTCGCGGAGCAGCTCCC CAACCCGGGTGCTCGGGCCCTGACCGTGCCGGATG

TSS sequence within promoter 2 (mm10 chr10:62865136-62865341)

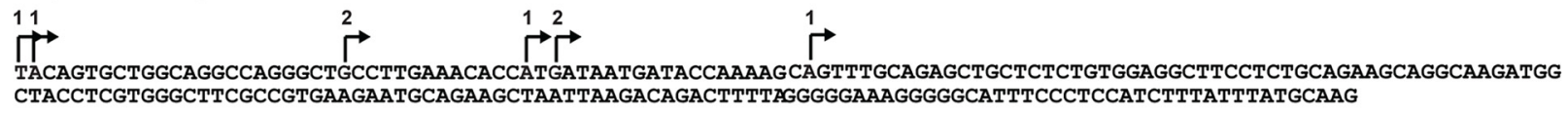

D

Revised Transcripts

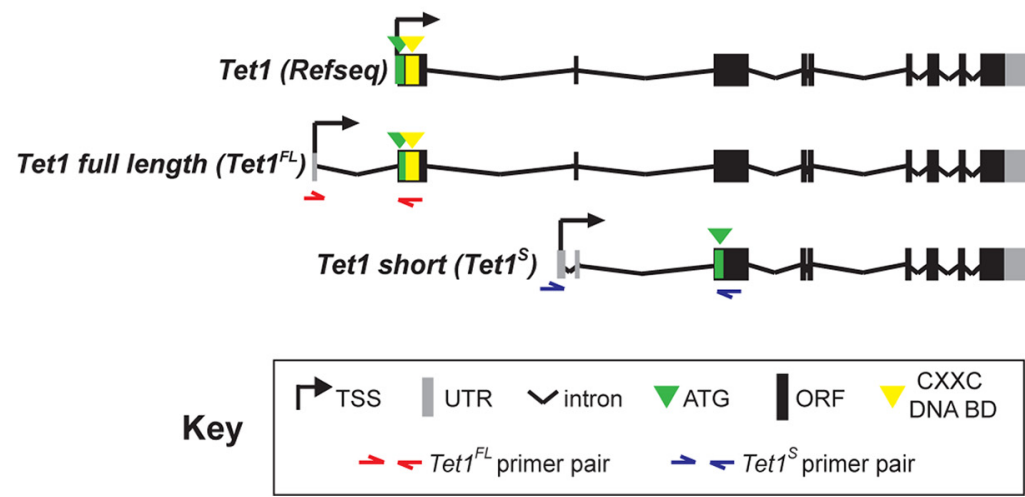

E

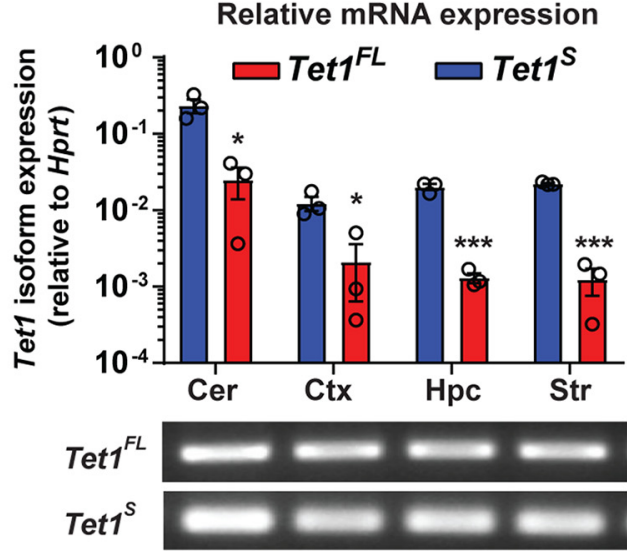

Figure 1. Tet1 is expressed as two distinct transcript isoforms in the adult mouse brain. A, Mean normalized H3K4me3, H3K27ac and H3K9ac ChIP-seq signal in NeuN+ hippocampal neurons at the Tet1 gene locus (Halder et al., 2016). Red and blue shaded areas depict putative Tet1 isoform promoter regions relative to the annotated RefSeq Tet1 transcript. Chr10 = Chromosome 10, Mbp = Mega base pairs. B, ChIP-qRT-PCR analysis of RNAP2 enrichment (\% input) at predicted Tet1 promoters in adult hippocampal tissue relative to a negative control (mouse Igx1a); $* p<0.05$ (Dunnett's post hoc test), $p<0.05$ (one-way ANOVA); $n=6$ mice. Data represent mean \pm SEM. $C$, 5' RACE sequence results summary from DNA clones amplified from adult hippocampal RNA; $n=8-10$ clones/isoform. Arrows and numbers above each nucleotide represent the TSSs and number of clones, respectively. $\boldsymbol{D}$, Illustration of revised Tet 1 isoform transcript architecture based on ChIP and 5' RACE data. Gray, untranslated region (UTR); black, Open Reading Frame (ORF); green, ATG (Translation Start Site); yellow, CXXC non-methyl (pG binding domain. Half arrows represent isoform specific primer locations. $\boldsymbol{E}$, top, qRT-PCR analysis of Tet 1 isoform expression levels in adult brain sub-regions relative to $H$ prt. Cer $=$ cerebellum, $\mathrm{Ctx}=$ cortex, Hpc = hippocampus, Str = striatum. Bottom, Image of endpoint PCR products generated after qRT-PCR using Tet $7^{F L}$ and Tet $7^{S}$-specific primers; $* p<0.05, * * * p<0.001$ (unpaired two-tailed $t$ test); $n=3$ per group. Data represent mean \pm SEM (Standard Error of the Mean).

marked by the repressive histone modification H3K27me3 (Fig. $2 \mathrm{C})$. The presence of active (H3K4me3 and $\mathrm{H} 3 \mathrm{~K} 9 \mathrm{ac})$ and repressive (H3K27me3) histone marks at Tet ${ }^{F L}$ exon 1 in neurons suggests that the Tet ${ }^{F L}$ promoter is bivalent in these cells, which has been shown to keep genes expressed at low basal levels, poised for reactivation (Bernstein et al., 2006). To test whether H3K27me3-mediated silencing accounted for differences in Tet ${ }^{F L}$ expression between glia and neurons, we blocked formation of the repressive mark using the two histone-lysine N-methyltransferase Enhancer of Zeste Homolog 2 (EZH2) inhibitors, GSK126 and GSK343 (Verma et al., 2012; Huang et al., 2019). As predicted by our chromatin analysis, both drugs significantly increased Tet $^{F L}$ transcript levels in neurons (GSK126: $F_{(2,12)}=8$, $p=0.0062$; two-way ANOVA; Tet $1^{F L}$ : DMSO, $1 \pm 0.15$ vs 1.25 $\mu \mathrm{M}, 1.8 \pm 0.18, p=0.011$, and DMSO, $1 \pm 0.15$ vs $2.5 \mu \mathrm{M}$, $2.6 \pm 0.31, p<0.001$; Dunnett's post hoc test; GSK343: $F_{(2,12)}=$ $4.3, p=0.039$; two-way ANOVA; Tet $1^{F L}$ : DMSO, $1 \pm 0.13$ vs 1.25 $\mu \mathrm{M}, 2.8 \pm 0.58, p=0.0062$, and DMSO, $1 \pm 0.13$ vs $2.5 \mu \mathrm{M}$, $3 \pm 0.6, p=0.0032 ; n=3$ all groups; Dunnett's post hoc test; Fig. $2 D, E$ ), while in glia its expression remained unchanged (GSK126: $F_{(2,12)}=0.69, p=0.52:$ GSK343: $F_{(2,12)}=0.91, p=0.43$; two-way ANOVA; Fig. 2F,G). Expression of Tet $^{S}$ was unaffected by the EZH2 inhibitors in either cell type (GSK126, neurons: DMSO, $1 \pm 0.10$ vs $1.25 \mu \mathrm{M}, 0.98 \pm 0.07, p=0.99$, and DMSO, $1 \pm 0.10$ vs $2.5 \mu \mathrm{M}, 0.71 \pm 0.03, p=0.4, n=3$ all groups; Dunnett's post hoc test; glia: DMSO, $1 \pm 0.03$ vs $1.25 \mu \mathrm{M}, 1.2 \pm 0.0091$, $p=0.15$, and DMSO, $1 \pm 0.030$ vs $2.5 \mu \mathrm{M}, 1.1 \pm 0.047, p=0.51$, $n=3$ all groups; Dunnett's post hoc test: GSK343, neurons: 
A

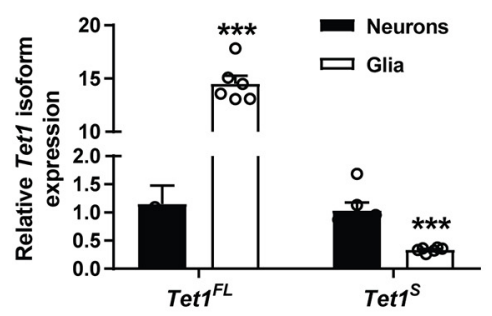

B

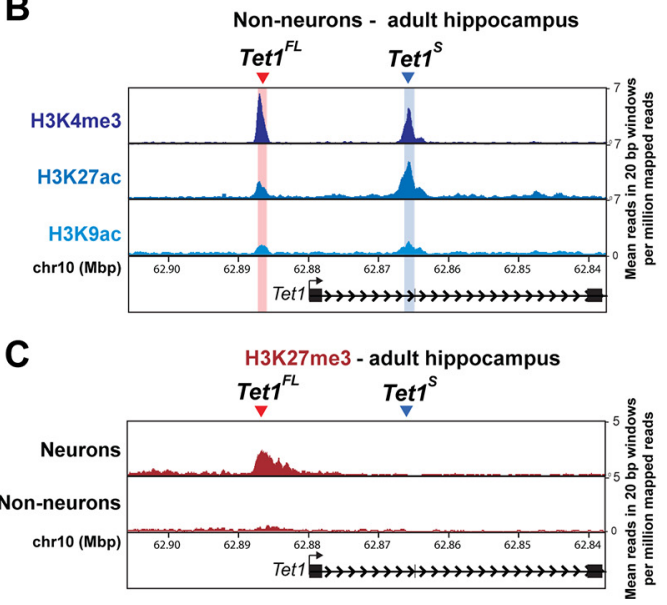

D

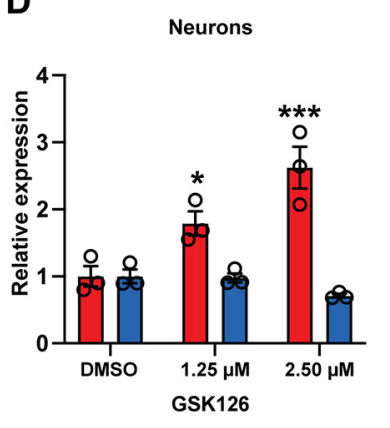

Glia

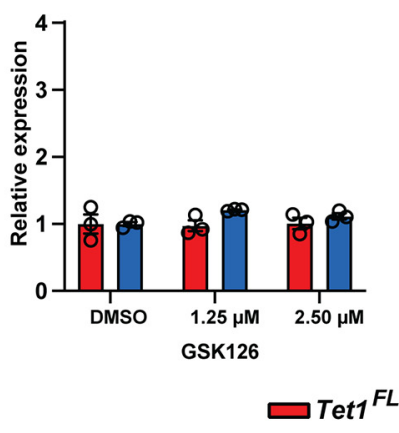

E

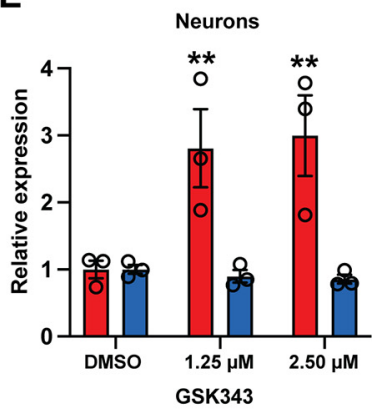

G

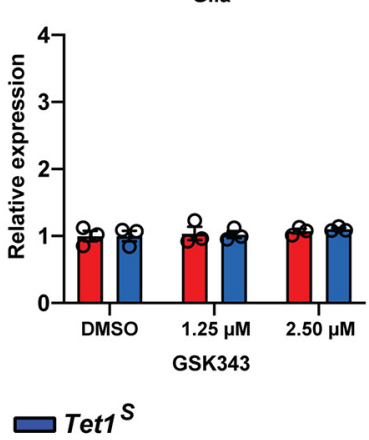

Figure 2. Tet1 isoform transcript usage differs between neurons and non-neuronal cells in the brain. $\boldsymbol{A}, \mathrm{qRT}-\mathrm{PCR}$ analysis of $T e t 7^{\mathrm{FL}}$ and Tet $1^{\mathrm{S}}$ isoform expression levels in primary hippocampal glial cultures relative to hippocampal neuron cultures; $* * * p<0.001$ (unpaired two-tailed $t$ test); $n=6$. B. Mean normalized H3K4me3, H3K27ac, and H3K9ac ChlP-seq signals in hippocampal non-neuronal cells (NeuN-) at the Tet1 gene locus. C, Comparison of mean normalized H3K27me3 ChlP-seq signals in hippocampal neurons (NeuN+) and non-neuronal (NeuN-) cells at the Tet1 gene locus. Data for both $\boldsymbol{B}, \boldsymbol{C}$ generated from Halder et al. (2016). D, E, qRT-PCR analysis of Tet $7^{F L}$ and Tet $7^{S}$ isoform expression levels in primary hippocampal neurons treated with the EZH2 inhibitors GSK126 and GSK343, respectively. F, G, qRT-PCR analysis of Tet ${ }^{F L}$ and Tet $1^{S}$ isoform expression levels in primary hippocampal glia treated with the EZH2 inhibitors GSK126 and GSK343, respectively. Seventy-two-hour treatments of indicated doses were conducted. Data normalized to vehicle (DMSO) for each isoform; $* p<0.05, * * p<0.01, * * * p<0.001$ (Dunnett's post hoc test vs vehicle); $p<0.01$ (two-way ANOVA for GSK126 in neurons), $p<0.05$ (two-way ANOVA for GSK343 in neurons); $n=3$ per group. All data represent mean \pm SEM.

DMSO, $1 \pm 0.07$ vs $1.25 \mu \mathrm{M}, 0.90 \pm 0.093, p=0.97$, and DMSO, $1 \pm 0.07$ vs $2.5 \mu \mathrm{M}, 0.85 \pm 0.07, p=0.94, n=3$ all groups; Dunnett's post hoc test; glia: DMSO, $1 \pm 0.08$ vs $1.25 \mu \mathrm{M}$, $1 \pm 0.052, p=0.96$, and DMSO, $1 \pm 0.08$ vs $2.5 \mu \mathrm{M}, 1.1 \pm 0.02$, $p=0.46, n=3$ all groups; Dunnett's post hoc test; Fig. $2 D-G)$. Together, these results demonstrate that Tet1 isoform transcript usage differs significantly between neurons and glia, and that Tet $1^{F L}$ expression is specifically suppressed in neurons through epigenetic mechanisms involving EZH2-generated H3K27me3 marks.

\section{Tet $1^{S}$ transcript levels are downregulated in response to neuronal activity}

We and others previously reported that total Tet 1 mRNA levels are decreased in response to neuronal activity (Kaas et al., 2013; Widagdo et al., 2014). To examine the contributions of the Tet ${ }^{F L}$ and $T e t 1^{S}$ transcripts to these changes, hippocampal neuron cultures were incubated for 1 or $4 \mathrm{~h}$ with $\mathrm{KCl}$, bicuculline, or NMDA/glycine, and expression levels were evaluated using qRTPCR. We found that Tet $1^{F L}$ mRNA levels were unaffected by any of these treatments (Tet $1^{F L}$ fold change, KCl: $F_{(2,45)}=0.6$, $p=0.55$; bic: $F_{(2,15)}=0.25, p=0.78$; NMDA/gly: $F_{(2,33)}=0.091$; one-way ANOVA; Fig. $3 A$ ). In contrast, Tet $^{S}$ mRNA levels were significantly decreased at both the 1 - and 4 -h time points after $\mathrm{KCl}$ stimulation $\left(\mathrm{Tet}^{S}\right.$ fold change: $F_{(2,45)}=15, p<0.0001$; oneway ANOVA; Veh, $1 \pm 0.034, n=16$ vs 1 h, $0.76 \pm 0.048, n=15$, $p<0.0001$, and Veh, $1 \pm 0.034, n=16$ vs $4 \mathrm{~h}, 0.75 \pm 0.024$, $n=16, p<0.0001$; Dunnett's post hoc test) and at the 4 -h time point after treatment with either bicuculline $\left(\operatorname{Tet} 1^{S}\right.$ fold change:
$F_{(2,15)}=15, p=0.0003$; one-way ANOVA; Veh, $1 \pm 0.048, n=6$ vs $4 \mathrm{~h}, 0.64 \pm 0.041, n=6, p=0.0002$; Dunnett's post hoc test) or NMDA/gly (fold change: $F_{(2,33)}=21, p<0.0001$; one-way ANOVA; Veh, $1 \pm 0.025, n=12$ vs $4 \mathrm{~h}, 0.58 \pm 0.045, n=12$, $p<0.0001$; Dunnett's post hoc test), suggesting that its expression is regulated by activity-dependent and NMDA receptor-dependent mechanisms (Fig. 3B). We confirmed that each of these treatments significantly increased expression levels of the immediate early gene (IEG) activity regulated cytoskeleton associated protein $(A r c)$, as expected (Arc fold change, $\mathrm{KCl}: F_{(2,44)}=6.034$, $p=0.0048$; one-way ANOVA; Veh, $1 \pm 0.063, n=16$ vs $1 \mathrm{~h}$, $3.3 \pm 0.4, n=15, p=0.04$, and Veh, $1 \pm 0.063, n=16$ vs $4 \mathrm{~h}$, $4.2 \pm 1.1, n=16, p=0.0030$; Dunnett's post hoc test: bic: $F_{(2,15)}=$ 113.4, $p<0.0001$; one-way ANOVA; Veh, $1 \pm 0.097, n=6$ vs 1 h, $7.1 \pm 0.4, n=6, p<0.0001$; Dunnett's post hoc test: NMDA/ gly: $F_{(2,33)}=27.02, p<0.0001$; one-way ANOVA; Veh, $1.1 \pm$ $0.064, n=12$ vs 1 h, $4.9 \pm 0.68, n=12, p<0.0001$; Dunnett's post hoc test; Fig. 3C).

Next, we measured $T e t 1^{F L}$ and $T e t 1^{S}$ expression levels in tissue extracted from hippocampal area CA1 after CxFC to examine whether both isoforms responded similarly to neuronal activity induced in vivo during memory formation. Again, we observed that $\operatorname{Tet}^{F L}$ transcript levels remained unchanged ( $\operatorname{Tet} 1^{F L}$ fold change: $F_{(2,25)}=0.2666, p=0.77$; oneway ANOVA; Fig. $3 D$ ), whereas Tet $1^{S}$ expression was significantly downregulated $1 \mathrm{~h}$ after training $\left(\operatorname{Tet}^{S}\right.$ fold change: $F_{(2,25)}=3.399, p=0.0494$; one-way ANOVA; hc, $1.1 \pm 0.17$, $n=9$ vs $1 \mathrm{~h}, 0.71 \pm 0.075, n=10, p=0.037$; Dunnett's post hoc test; Fig. $3 E)$. As in primary cultures, $\operatorname{Arc}$ expression was 
significantly induced in CA1 after CxFC (Arc fold change: $F_{(2,25)}=4.55, p=0.021$, one-way ANOVA; hc, $1.2 \pm 0.25, n=9$ vs 1 h, $2.5 \pm 0.44, n=10, p=0.015$; Dunnett's post hoc test; Fig. $3 F$ ). Together, these results show that transcript levels of the Tet ${ }^{S}$ gene are generally downregulated in response to hippocampal neuron stimulation, suggesting that under basal conditions the isoform may act as a molecular restraint on activity-dependent processes in neurons.

Individual manipulation of Tet $^{F L}$ and Tet1 $^{S}$ expression in hippocampal neurons The differential regulation of Tet1 isoform expression following neuronal activation suggests that $\operatorname{Tet}^{F L}$ and $\operatorname{Tet}^{S}$ may have unique cell-specific functions. However, prior studies globally manipulated the expression of both genes, providing limited insight into the cell-specific functions of each Tet1 isoform. Therefore, we developed genetic tools to selectively manipulate $\operatorname{Tet}^{\mathrm{FL}}$ and $\operatorname{Tet}^{S}$ expression levels in neurons, both in culture and in vivo. To accomplish this, we designed sequenceprogrammable TALEs to selectively target Tet $1^{\text {FL }}$ or $T e t 1^{S}$ because of their previously reported high target specificity, cell type-specific expression, and small size compatible with in vivo delivery using a single AAV virus (Konermann et al., 2013; Mendenhall et al., 2013; Juillerat et al., 2014; Polstein et al., 2015). HA-tagged TALEs were designed to specifically bind to DNA sequences at each Tet1 isoform promoter and either repress transcription (TALE-SID4X, four copies of the $\mathrm{mSin} 3$ interacting domain) or serve as a target sequence-specific control (TALE-NFD, no functional domain; Konermann et al., 2013; Choi et al., 2014; Fig. 4A,B). TALE expression in these modified AAV vectors was placed under control of the human synapsin I promoter (hSYN), which drives expression only in neurons (Kügler et al., 2003). We found that expression of either control TALE in N2a cells, hereafter referred to as Tet $1^{F L}$-NFD and $T e t 1^{S}$-NFD, did not alter the expression of the Tet 1 isoforms relative to mock-transfected cells, suggesting that TALE binding to these promoter regions without an effector domain did not sterically hinder transcription (fold changes: mock, $1 \pm 0.095$ vs Tet $1^{F L}$-NFD, $1 \pm 0.061, t_{(6)}=0.069, p=0.95$, and mock, $1 \pm 0.053$ vs Tet $^{S}$-NFD, $1.1 \pm 0.067, t_{(6)}=0.68, p=0.52, n=4$ all groups; unpaired two-tailed $t$ tests; Fig. $4 C, D)$. Conversely, expression of the TALE repressors, hereafter referred to as Tet $1^{F L}$-SID4X and $T e t 1^{S}$-SID4X, significantly inhibited the expression of $\operatorname{Tet}^{F L}$ and $\operatorname{Tet}^{S}$ in $\mathrm{N} 2 \mathrm{a}$ cells, respectively. Importantly, neither TALE repressor affected transcript levels of the opposite isoform, indicating isoform-specific targeting (Tet $1^{F L}$ fold changes: $F_{(2,9)}=26, p=0.0002$; one-way ANOVA; Tet $1^{F L}$-NFD, $1.1 \pm 0.044$ vs $\operatorname{Tet}^{F L}$-SID $4 \mathrm{X}, 0.42 \pm 0.021$, $p=0.0006$, and $\operatorname{Tet}^{F L}{ }^{\text {-NFD, }} 1.1 \pm 0.044$ vs Tet ${ }^{S}$-SID4X, $1.2 \pm$ $0.14, p=0.57, n=4$ all groups; Dunnett's post hoc test: Tet $1^{S}$ fold changes: $F_{(2,9)}=17, p=0.0008$; one-way ANOVA; Tet $1^{S}$-NFD, $1 \pm 0.061$ vs Tet $^{S}$-SID4X, $0.15 \pm 0.0039, p=0.0009$, and Tet $1^{S}$-NFD, $1 \pm 0.061$ vs Tet $1^{\text {FL }}$-SID $4 X, 0.91 \pm 0.18, p=0.78$, $n=4$ all groups; Dunnett's post hoc test; Fig. $4 E, F)$. In addition, we measured the effects of Tet $1^{F L}$-SID $4 \mathrm{X}$ and $T e t 1^{S}$-SID4X on the expression of any predicted off-target genes that contained either TALE sequence up to three mismatches within +1000 to -400 bases of its TSS. Genes identified as TET1 targets in the ChIP enrichment analysis (ChEA) transcription factor targets dataset were excluded from the analysis (Lachmann et al., 2010). We found that expression of either TALE repressor did not result in any significant changes in the transcript levels of their respective off-target genes, providing further evidence of their specificity (Fig. 4G,H; Extended Data Figs. 4-1, 4-2). Next, to examine the function of the Tet 1 isoform-specific TALEs in primary cells, we packaged each construct into AAV1 viral particles and transduced hippocampal neurons to investigate their efficacy and specificity. Immunocytochemistry revealed that 
A
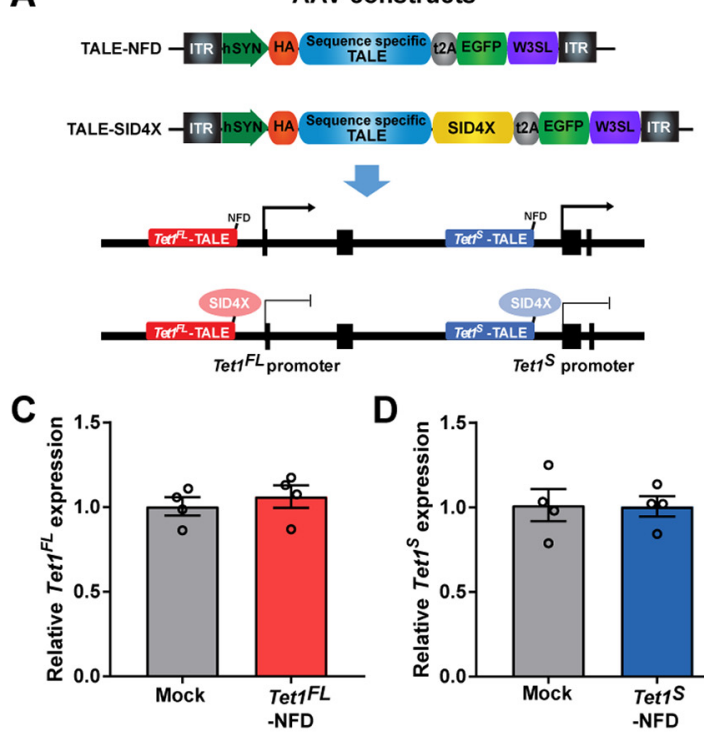

G

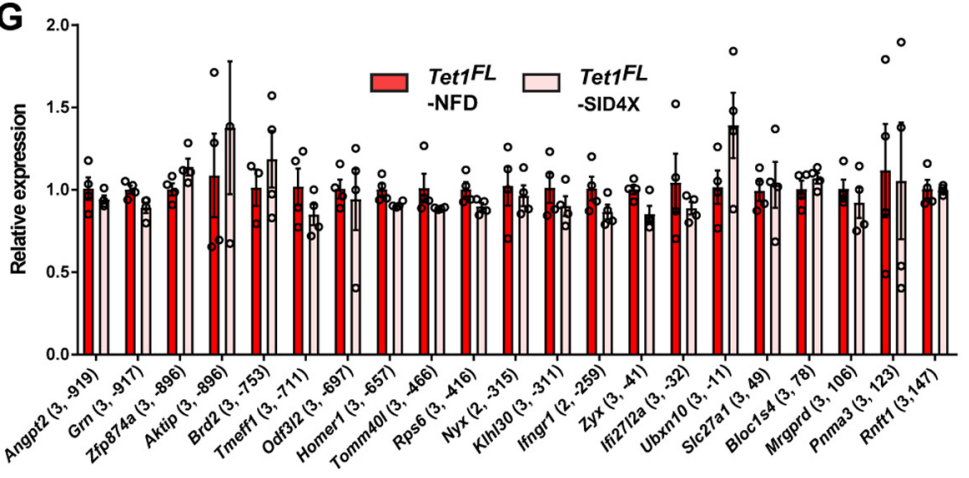

Predicted off-target genes

\section{I}
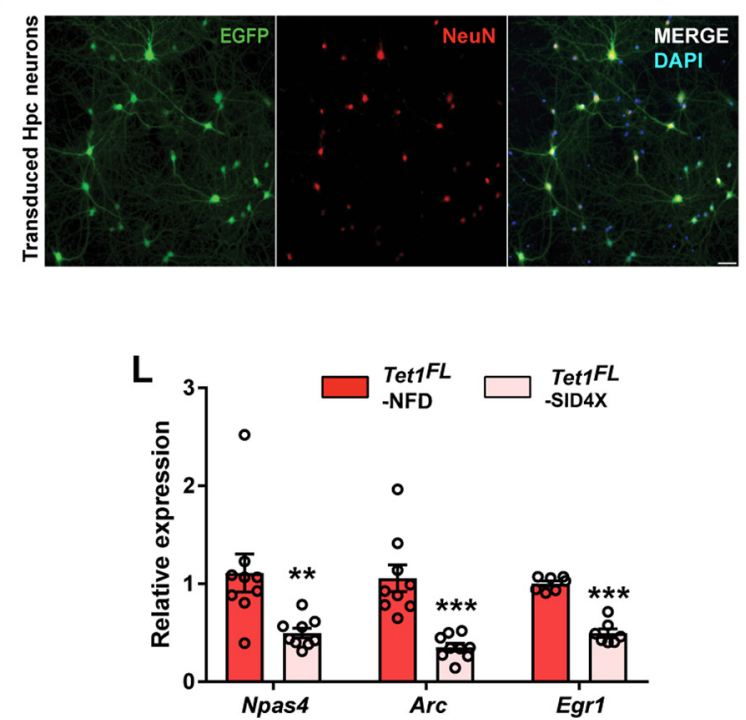

M
B

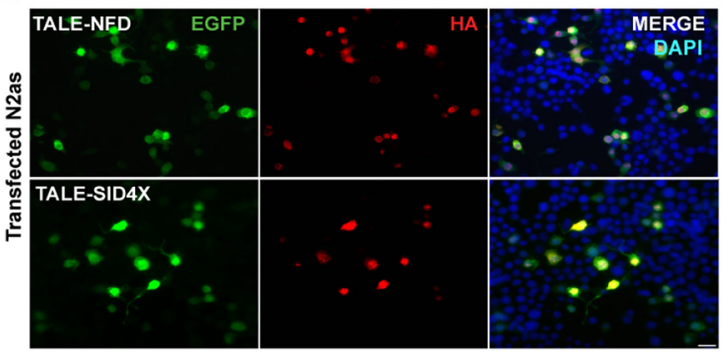

E
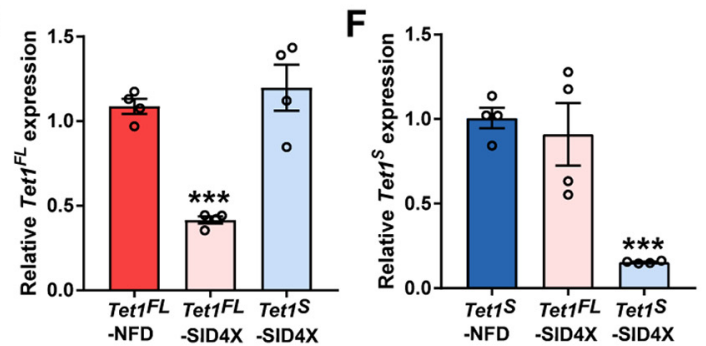

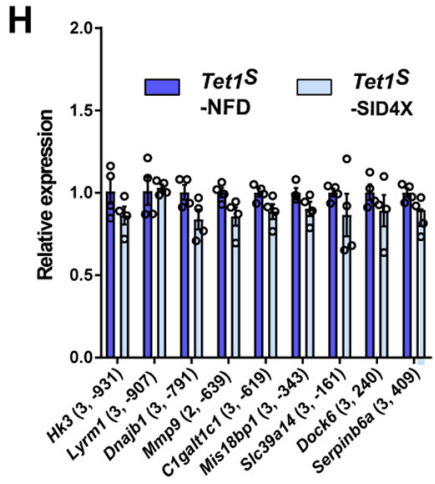

Predicted off-target genes

K

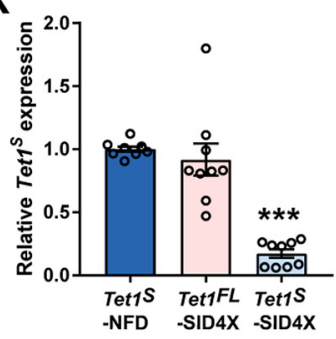

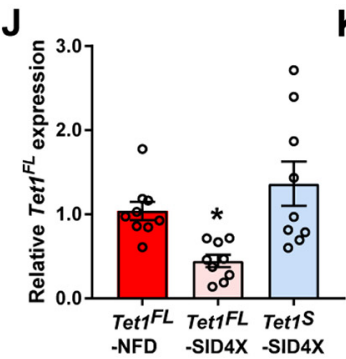

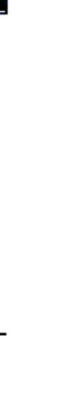


expression of the TALE constructs was neuron specific, as only cells positive for the neuronal marker NeuN expressed EGFP (Fig. 4I). Similar to their effects in N2a cells, transduction of primary hippocampal neurons with AAV1-Tet1 ${ }^{F L}$-SID4X and Tet $1^{S}$-SID4X led to a significant reduction in the expression levels of their intended Tet1 isoform target without affecting the opposite transcript (Tet $1^{F L}$ fold changes: $F_{(2,24)}=7.549$, $p=0.0029$; one-way ANOVA; Tet $1^{F L}$-NFD, $1 \pm 0.11$ vs $T e t 1^{F L}$ SID4X, $0.45 \pm 0.073, p=0.039$, and Tet $1^{F L}$-NFD, $1 \pm 0.11$ vs Tet ${ }^{S}$-SID4X, 1.4 $\pm 0.26, p=0.31, n=9$ all groups; Dunnett's post hoc test: Tet $1^{S}$ fold changes: $F_{(2,24)}=35, p<0.0001$; oneway ANOVA; Tet $1^{S}$-NFD, $1 \pm 0.02$ vs Tet $1^{S}$-SID4X, $0.17 \pm$ $0.033, p<0.0001$, and $T e t 1^{S}$-NFD, $1 \pm 0.02$ vs Tet ${ }^{\text {FL }}$-SID4X, $0.92 \pm 0.13, p=0.66, n=9$ all groups; Dunnett's post hoc test; Fig. $4 J, K)$. Finally, we measured transcript levels of the IEGs Npas4, Arc, and Egr1 in primary hippocampal neurons transduced with AAV1-Tet $1^{F L}$-SID4X or Tet $1^{S}$-SID4X because TET1 was previously shown to regulate their expression in the brain (Kaas et al., 2013; Rudenko et al., 2013; Kumar et al., 2015; Towers et al., 2018). We found that SID4X-mediated repression of either Tet1 isoform led to a significant reduction in the expression of all three genes compared with NFD controls (Tet $1^{F L}$-NFD vs Tet $1^{F L}$-SID4X fold changes: Npas $4,1.1 \pm 0.19$ vs $0.38 \pm 0.041$, $t_{(16)}=3.7, n=9, p=0.002 ; A r c, 1.1 \pm 0.14$, vs $0.35 \pm 0.04, t_{(16)}=$ $5, n=9, p<0.0001 ; E g r 1,1 \pm 0.027$, vs $0.5 \pm 0.043, t_{(12)}=10$, $n=7, p<0.0001$ : Tet $^{S}$-NFD vs Tet $^{S}$-SID $4 \mathrm{X}$ fold changes: Npas4, $1.1 \pm 0.22$ vs $0.32 \pm 0.057 t_{(16)}=3.6, n=9, p=0.0023$; Arc, $1.2 \pm 0.23$ vs $0.51 \pm 0.12, t_{(16)}=2.6, n=9, p=0.021$; Egrl, $1 \pm 0.032$ vs $0.35 \pm 0.014, t_{(12)}=19, n=7, p<0.0001$; unpaired two tailed $t$ tests; Fig. $4 L, M$ ). Together, these results demonstrate that our modified TALE tools significantly repress the transcription of each individual Tet 1 isoform, are neuron specific, and result in changes in the expression of genes previously shown to be targets of TET1 in the CNS.

\section{Tet $1^{F L}$ and $\operatorname{Tet}^{S}$ regulate unique subsets of the neuronal transcriptome}

To investigate the effects of each individual Tet1 isoform on neuronal gene expression, we infected postnatal hippocampal neurons with our AAV1-Tet $1^{F L}$-SID4X, -Tet $1^{F L}$-NFD, - Tet $^{S}$ SID4X, or $-\operatorname{Tet}^{S}$-NFD and performed an unbiased, transcriptomewide RNA-seq analysis on control and Tet1 isoform-depleted cells. We found that despite its low transcript levels, acute repression

of predicted off-target genes for the Tet ${ }^{F L}$ TALE target sequence. ( $x$-axis key: \# mismatches, distance relative to TSS); $n=3-4$ per group. Statistical outcomes in Extended Data Figure 41. $\boldsymbol{H}$, qRT-PCR analysis of predicted off-target genes for the Tet ${ }^{5}$ TALE target sequence $(x-$ axis key: \# mismatches, distance relative to TSS); $n=3-4$ per group. Statistical outcomes in Extended Data Figure 4-2. I, Representative EGFP and NeuN immunostaining of primary hippocampal cultures $5 \mathrm{~d}$ after transduction with AAV1-TALE constructs. Scale bar: $50 \mu \mathrm{m}$. $J$, qRT-PCR analysis of Tet $7^{F L}$ expression levels in primary hippocampal neurons transduced with AAV1-Tet7 ${ }^{F L}$-NFD, -Tet7 $1^{F L}$-SID4X, or -Tet7 ${ }^{S}$-SID4X; $* p<0.05$ (Dunnett's post hoc test); $p<0.05$ (one-way ANOVA); $n=9$ per group. $\boldsymbol{K}$, qRT-PCR analysis of $\operatorname{Tet}^{\mathrm{S}}$ expression levels

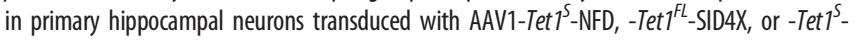
SID4X; $* * * p<0.001$ (Dunnett's post hoc test); $p<0.05$ (one-way ANOVA); $n=9$ per group. L, qRT-PCR analysis of Npas4, Arc, and Egr1 expression levels in primary hippocampal neurons transduced with AAV1-Tet7 ${ }^{F L}$-NFD and -Tet7 ${ }^{F L}$-SID4X; $* * p<0.01, * * * p<0.001$ (unpaired two-tailed $t$ test); $n=9$ per group. M, qRT-PCR analysis of Npas4, Arc, and Egr1 expression levels in primary hippocampal neurons transduced with AAV1-Tet $1^{5}$-NFD and -Tet $T^{5}$-SID4X; $* p<0.05, * * p<0.01, * * * p<0.001$ (unpaired two-tailed $t$ test); $n=9$ per group. All data represent mean \pm SEM. of $T e t 1^{F L}$ caused widespread transcriptional changes in neurons. Using a cutoff greater than $\pm 0.2 \log 2$ fold-change $(\log 2 \mathrm{FC})$ and an FDR $<0.05$, we identified $>6000$ differentially expressed genes (DEGs; Fig. 5A, top; Extended Data Fig. 5-1). Gene Ontology (GO) analysis revealed that $\operatorname{Tet}^{F L}$-modulated mRNAs functioned in a wide assortment of biological processes (BP) and Kyoto Encyclopedia of Genes and Genomes pathways (KEGG; Fig. 5A, bottom; Extended Data Figs. 5-2, 5-3). In both sets of analyses, genes downregulated in response to Tet $1^{F L}$ repression were generally enriched for neuron-associated functions, such as ion transport, learning, long-term synaptic potentiation and the synaptic vesicle cycle (BP terms and KEGG terms: ion transport: $3.3 \times 10^{-20}$, learning: $3.6 \times 10^{-12}$, long-term synaptic potentiation: $7.8 \times 10^{-8}$, synaptic vesicle cycle: $1.2 \times 10^{-12}$; Benjamini-Hochberg adjusted $p$ values; Fig. $5 A$, bottom left). In contrast, upregulated genes were enriched for BP terms associated with the cell cycle, DNA damage, and immune function $\left(6.3 \times 10^{-25}, 1.6 \times 10^{-14}, 1.3 \times 10^{-9}\right.$, respectively; Benjamini-Hochberg adjusted $p$ values) as well as several KEGG pathway categories related to cancer pathways, extracellular matrix (ECM) receptor interactions, and NF- $\kappa \mathrm{B}$ signaling $\left(3.5 \times 10^{-15}, 1.1 \times 10^{-11}, 7.3 \times 10^{-10}\right.$, respectively; Benjamini-Hochberg adjusted $p$ values; Fig. $5 A$, bottom right). In the case of $T e t 1^{S}$, we found that its repression led to less than a quarter of the number of DEGs relative to Tet $1^{F L}$ (Fig. 5B, top; Extended Data Fig. 5-4). Genes upregulated following Tet $1^{S}$ repression were enriched for some of the same BP categories as $\operatorname{Tet}^{F L}$, but also included terms associated with ribosomal biogenesis, methylation, and covalent chromatin modifications $\left(5.1 \times 10^{-4}, 2 \times 10^{-3}, 3.7 \times 10^{-3}\right.$, respectively; BenjaminiHochberg adjusted $p$ values; Fig. $5 B$, bottom right; Extended Data Fig. 5-5). Notably, downregulated genes associated with $T e t 1^{S}$ were not significantly enriched in any pathway or GO category (Extended Data Fig. 5-6). To further explore differences between $T e t 1^{F L}$ and $T e t 1^{S}$-mediated transcriptional regulation, we performed a direct comparison of Tet $1^{S}$-SID4X to Tet ${ }^{F L}$ SID4X associated DEGs. Overall, we found that differentially expressed genes altered in response to the loss of each isoform did not entirely overlap, suggesting the two Tet 1 isoforms do not serve redundant functions in neurons. Slightly over half of activated genes (Fig. 5C; Extended Data Fig. 5-7) and repressed genes (Fig. 5D; Extended Data Fig. 5-7) after modulation of the short isoform were also changed after suppression of the long isoform. This prompted us to do a direct comparison of the SID4X conditions, and this further statistical analysis (Extended Data Fig. 5-8) revealed that DEGs expressed at lower levels in the $T e t 1^{S}$-SID4X dataset, relative to Tet ${ }^{F L}$-SID4X, were generally involved in immune system regulation (BP terms; Immune system process: $1.7 \times 10^{-17}$, inflammatory response: 2.2 $\times 10^{-13}$, innate immune response: $1.6 \times 10^{-9}:$ KEGG terms; TNF signaling pathway: $4.9 \times 10^{-8}$, Nf- $\kappa \mathrm{B}$ signaling pathway: $6.5-$ $\times 10^{-8}$; Benjamini-Hochberg adjusted $p$ values; Fig. $5 E$, bottom left; Extended Data Fig. 5-9). Genes more abundantly expressed when $\operatorname{Tet}^{S}$ was repressed relative to $\operatorname{Tet}^{F L}$, functioned in transport, regulation of synaptic plasticity, and learning $\left(1.2 \times 10^{-2}, 3.3\right.$ $\times 10^{-2}, 3.7 \times 10^{-2}$, respectively; Benjamini-Hochberg adjusted $p$ values; Fig. 5E, bottom right; Extended Data Fig. 5-10). We interpret this to mean that while overlaps exist, the acute repression of Tet ${ }^{F L}$ aberrantly activates inflammatory response pathways, while $\operatorname{Tet}^{S}$ does not. Moreover, relative to $T e t 1^{F L}$, the acute suppression of $\operatorname{Tet}^{S}$ elicits higher expression of genes involved in synaptic plasticity. 
A

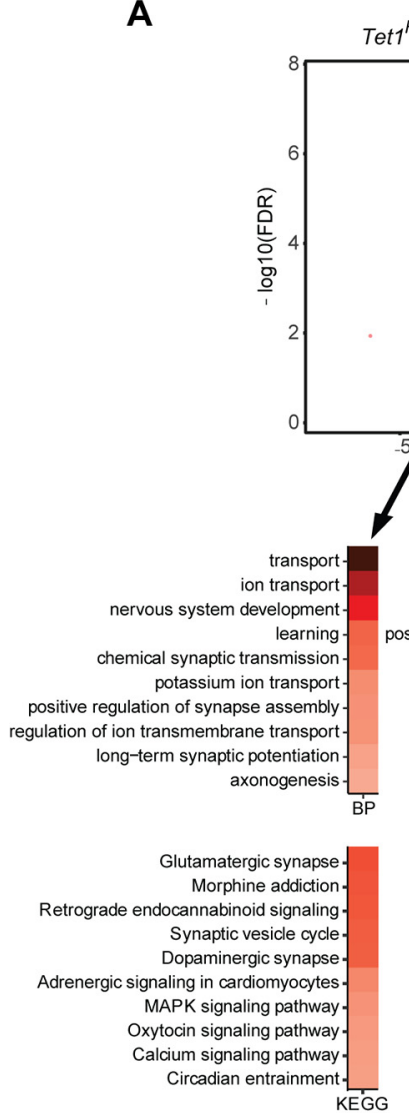

Tet1 $^{F L_{-} \text {-SID4X vs. Tet1 }}{ }^{F L_{\text {-NFD }}}$

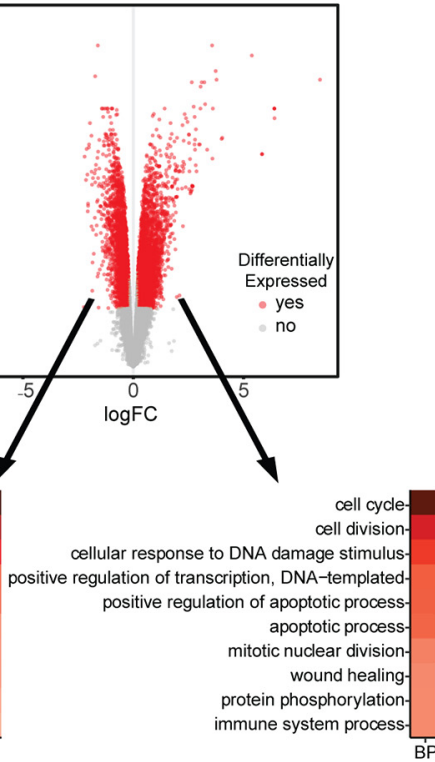

\section{C}

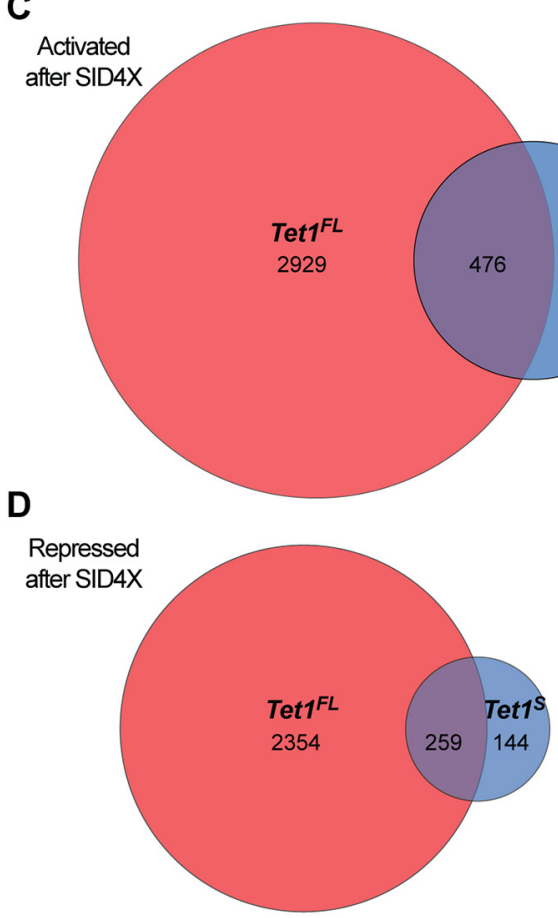

p53 signaling pathway.

Small cell lung cancer. ECM-recptor interaction HTLV-I infection NF-kappa B signaling pathwayApoptosisHippo signaling pathway.
B

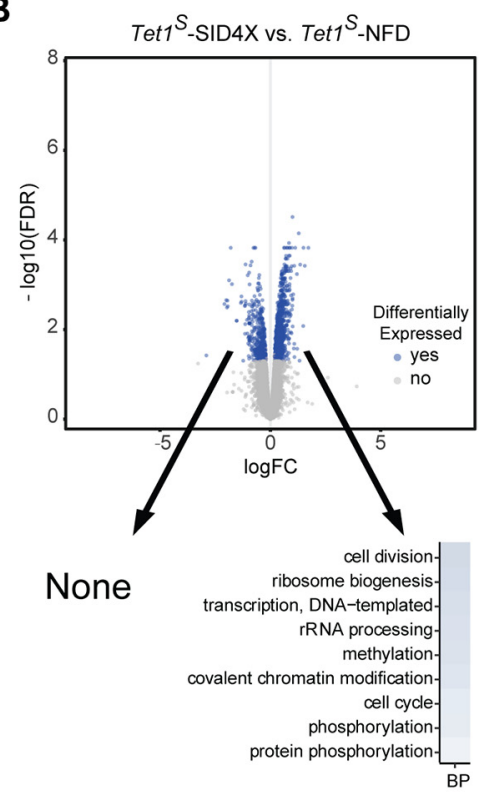

None Ribosome biogenesis in eukaryotes-

$$
\begin{gathered}
-\log 10 \text { (pvalue) } \\
081624
\end{gathered}
$$

\section{E}

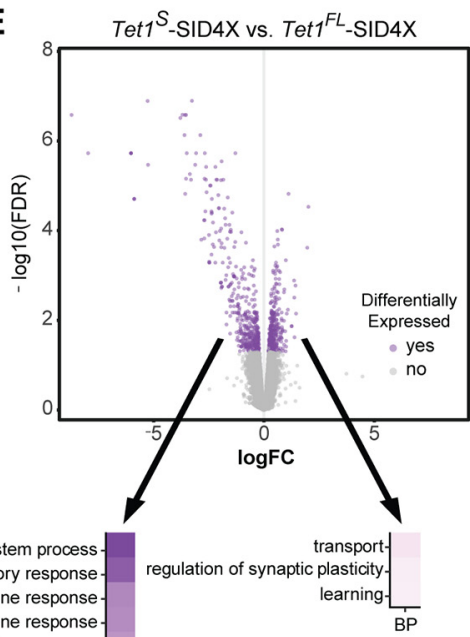

cellular response to interferon-gamma response to lipopolysaccharide.

defense response to Gram-positive bacterium lipopolysaccharide-mediated signaling pathway. cellular response to lipopolysaccharide cellular response to interleukin-1

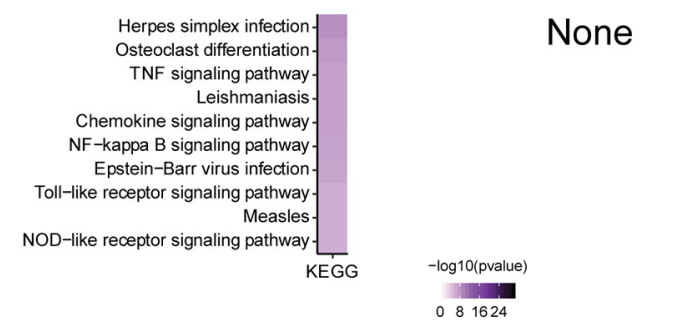

Figure 5. Transcriptomic analysis of hippocampal neuron cultures depleted of Tet $7^{F L}$ and $T e t 7^{S}$. $\boldsymbol{A}$, Top, Volcano plot analysis of genome wide RNA-seq data comparing AAV1-Tet $7^{F L}$-NFD and Tet7 ${ }^{F L}$-SID4X transduced hippocampal neurons $( \pm 0.2 \log 2 \mathrm{FC}$, FDR $<0.05) ; n=3$ biological replicates. Extended Data Figure 5-1, all genes; Extended Data Figures 5-2, 5-3, downregulated and upregulated DEGs, respectively. Bottom, Ten most statistically significant BP and KEGG enrichment terms associated with upregulated and downregulated DEGs in Tet $7^{F L}$-SID4X versus Tet $7^{F L}$ NFD transduced hippocampal neurons; $p<0.05$ (Benjamini-Hochberg corrections). Extended Data Figures 5-2, 5-3, all BP and KEGG terms associated with downregulated and upregulated DEGS, respectively. $\boldsymbol{B}$, Volcano plot analysis of genome wide RNA-seq data comparing AAV1-Tett $T^{5}$-NFD and -Tet $7^{5}$-SID4X transduced hippocampal neurons $( \pm 0.2 \log 2 \mathrm{FC}, \mathrm{FDR}<0.05) ; n=3$ biological replicates. Extended Data Figure 5-4, all genes; Extended Data Figures 5-5, 5-6, downregulated and upregulated DEGs, respectively. Bottom, Statistically significant BP and KEGG 
Acute repression of Tet $1^{F L}$ and Tet $1^{S}$ expression has opposing effects on synaptic transmission

Prior studies have found that Tet1 KO animals do not exhibit altered hippocampal long-term potentiation (LTP; Rudenko et al., 2013; Kumar et al., 2015; Towers et al., 2018). However, shRNA-mediated KD of Tet 1 in neuron cultures has been shown to increase mEPSCs amplitudes, suggesting that the gene regulates at least some aspects of synaptic transmission (Yu et al., 2015). To examine whether Tet $1^{F L}$, or $\operatorname{Tet}_{1}{ }^{S}$, might be responsible for these previously reported electrophysiological changes, we transduced hippocampal cultures with AAV1TALEs using the same conditions as in Figure $4 J, K$ and recorded mEPSCs in EGFP+ control and Tet 1 isoform-depleted neurons. We found that SID4X-mediated repression of Tet $1^{F L}$ significantly increased both mEPSC amplitude and frequency compared with controls (Fig. $6 A-C)$, as reflected by significant rightward shifts in the cumulative probability distributions of both measurements (amplitude: $D=$ $0.10, p=0.012$, frequency: $D=0.23, p<0.0001$, Tet $^{F L}$-NFD,$n=19$, Tet $^{F L}$-SID4X, $n=20$; Kolmogorov-Smirnov test). In contrast, Tet $1^{S}$ repression had no effect on mEPSC amplitude (Fig. 6D,E), but significantly reduced mEPSC frequency (Fig. $6 D, F$ ), illustrated by a significant leftward shift in the cumulative probability distribution (amplitude: $D=0.066, p=0.15$, frequency: $D=0.32, p<0.0001$, Tet $1^{S}$-NFD, $n=20, \quad$ Tet $1^{S}$-SID4X, $n=23$; KolmogorovSmirnov test). Overall, these data suggest that the acute and selective repression of Tet1 isoform expression differentially regulates excitatory synaptic transmission.

\section{$\operatorname{Tet1}^{F L}$ and Tet $^{S}$ differentially regulate hippocampal-dependent memory}

We next assessed the cognitive effects of selectively inhibiting $\operatorname{Tet}^{F L}$ and $T e t 1^{S}$ expression in the dorsal hippocampus (dHPC) using our neuron-specific and isoform-specific molecular tools. Stereotaxic injection of Tet1 isoform-specific AAV1-TALEs into the dHPC led to widespread

$\leftarrow$

enrichment terms of upregulated DEGs in AAV1-Tet $T^{5}$-SID4X versus -Tet $T^{5}$-NFD transduced hippocampal neurons. None, no statistically significant G0 terms identified; $p<0.05$ (Benjamini-Hochberg corrections). Extended Data Figures 5-5, 5-6, all BP and KEGG terms associated with downregulated and upregulated DEGs, respectively. C, D, Overlapping genes upregulated and downregulated, respectively, in AAV1-Tet $7^{S}$-SID4X versus - Tet $^{F^{L}}$-SID4X transduced hippocampal neurons. Extended Data Figure 5-7, lists of overlapping downregulated and upregulated DEGs. $\boldsymbol{E}$, top, Volcano plot analysis of genome wide RNA-seq data comparing AAV1-Tet $T^{S}$-SID4X and - Tet $^{F L}$-SID4X transduced hippocampal neurons $( \pm 0.2$ $\log 2 \mathrm{FC}, \mathrm{FDR}<0.05) ; n=3$ biological replicates. Extended Data Figure 5-8, all genes; Extended Data Figures 5-9, 5-10, downregulated and upregulated DEGs, respectively. Bottom, Ten most statistically significant BP and KEGG enrichment terms associated with upregulated and downregulated DEGs in AAV1-Tet7 ${ }^{5}$-SID4X vs - Tet7 ${ }^{\text {FL }}$-SID4X transduced hippocampal neurons; $p<0.05$ (Benjamini-Hochberg corrections). Extended Data Figures 5-9, $5-10$, all BP and KEGG terms associated with downregulated and upregulated DEGs, respectively.
D

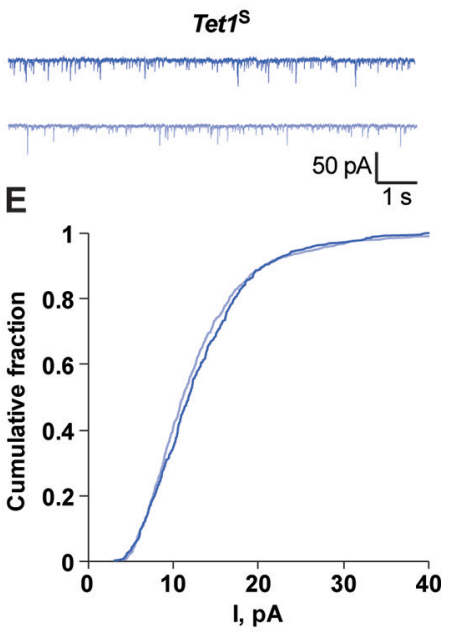

$\mathbf{F}$

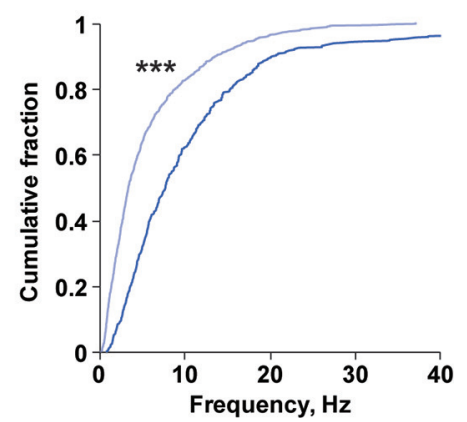

Figure 6. $\quad \operatorname{Tet}^{F L}$ and $\operatorname{Tet}^{S}$ repression alters excitatory synaptic transmission. $\boldsymbol{A}$, Representative mEPSC traces from hippocampal neurons transduced with AAV1-Tet ${ }^{F L}$-NFD and $-T e t 1^{F L}-S I D 4 X$, calibration: $1 \mathrm{~s}, 50 \mathrm{pA}$. $\boldsymbol{B}$, Cumulative probability distribution plots of mEPSC amplitudes in hippocampal neurons transduced with AAV1-Tet $7^{F L}$-NFD and *** $<0.001$ (Kolmogorov-Smirnov test); $n=19-20$ cells per group. $\boldsymbol{D}$, Representative mEPSC traces from hippo列 0.05 (Kolmogorov-Smirnov test); $n=20-23$ cells per group. $\boldsymbol{F}$, Cumulative probability distribution plots of mEPSC frequencies in hippocampal neurons transduced with AAV1-Tet1 ${ }^{F L}$-NFD and - Tet $^{F L}$-SID4X; $* * * p<0.001$ (Kolmogorov-Smirnov test); $n=20-23$ cells per group.

Enhanced Green Fluorescent Protein (EGFP) expression throughout CA1-CA3 subfields after two weeks (Fig. 7A). Importantly, and similar to our findings in vitro, hippocampal transduction with AAV1-Tet $1^{F L}$-SID4X and -Tet ${ }^{S}$-SID4X lead to significant reductions in $T e t 1^{F L}$ and $T e t 1^{S}$, respectively, without affecting the opposite isoform, suggesting that these molecular tools were also effective in vivo (Tet $1^{F L}$ fold changes: $F_{(2,21)}=3.49$, $p=0.049$; one-way ANOVA; Tet $1^{F L}-\mathrm{NFD}, 1.1 \pm 0.13$ vs Tet $^{F L}{ }_{-}$ SID4X, 0.73 $\pm 0.051, p=0.028$, and Tet $1^{F L}-\mathrm{NFD}, 1.1 \pm 0.13$ vs Tet1 ${ }^{S}$-SID4X, $0.91 \pm 0.096, p=0.31, n=8$ all groups; Dunnett's post hoc test: Tet $1^{S}$ fold changes: $F_{(2,21)}=18.45, p<0.0001$; one-way ANOVA; Tet $1^{S}$-NFD, $1.05 \pm 0.063$ vs Tet $1^{S}$-SID4X, $0.57 \pm 0.044$, $p<0.0001$, and Tet $^{S}$-NFD, $1.05 \pm 0.063$ vs Tet ${ }^{F L}$-SID4X, $0.92 \pm 0.0 .064, p=0.25, n=8$ all groups; Dunnett's post hoc test; Fig. $7 B, C)$.

We first tested whether transcriptional repression of Tet $1^{F L}$ and $\mathrm{Tet}^{S}$ in the dHPC led to any changes in locomotion or anxiety. In both the open field and Elevated Zero Maze (EZM) tests, there were no significant differences between mice infected with virus to silence either isoform compared with their isoformspecific controls [open field, total distance $(\mathrm{cm}): \operatorname{Tet}^{F L}-\mathrm{NFD}$, 
A
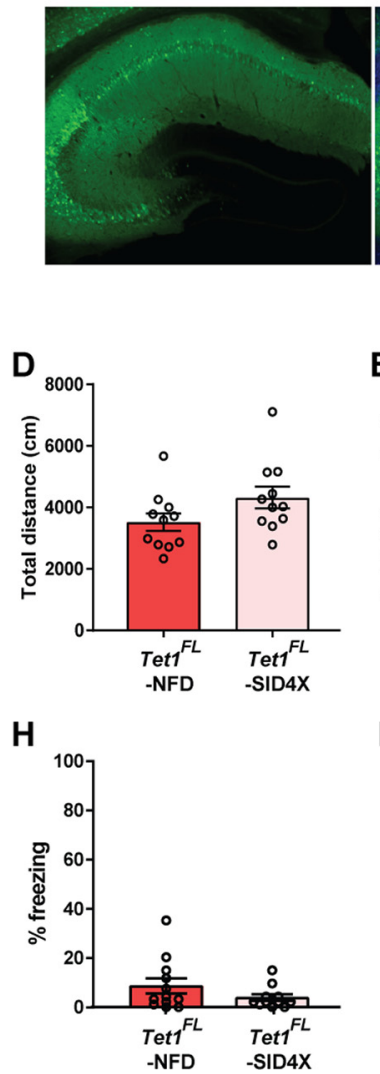

B

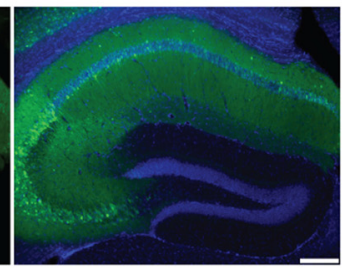

E

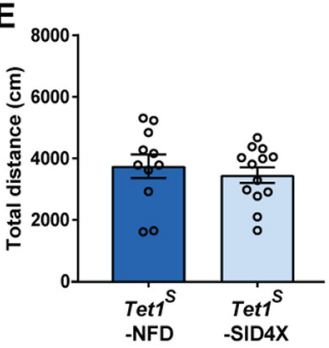

I

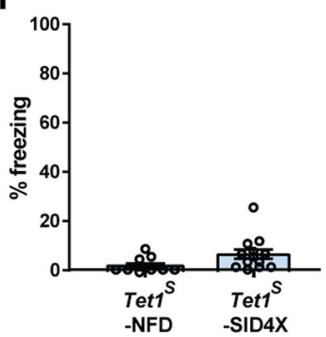

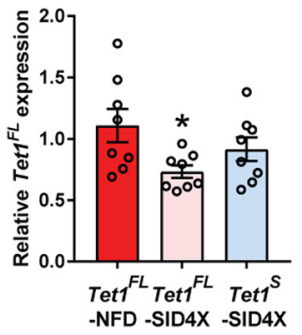

F

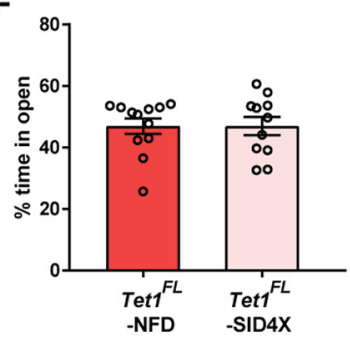

J

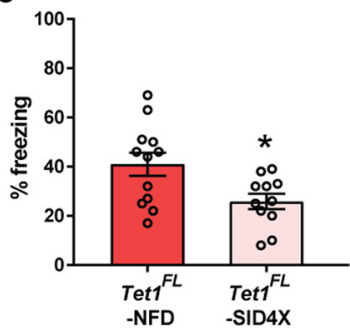

C

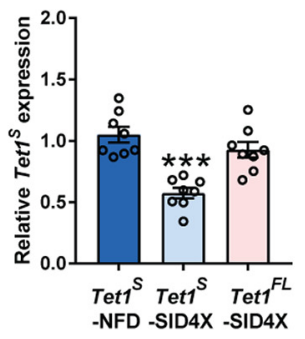

G

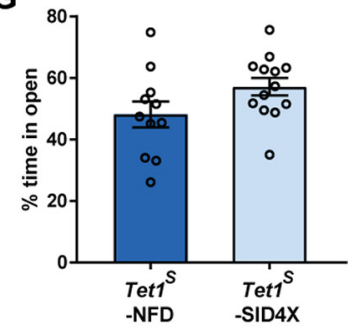

K

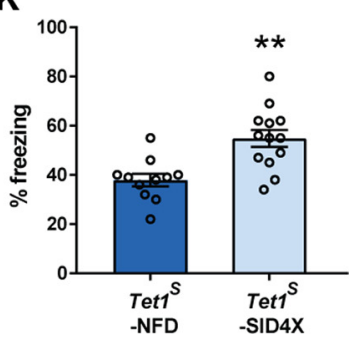

Figure 7. Neuron-specific Tet $7^{F L}$ and $T e t T^{S}$ repression differentially affect hippocampal-dependent memory formation. $A$, left, Representative EGFP immunostaining in the dHPC two weeks after AAV1-mediated transduction of Tet1 isoform-specific TALE constructs. Right, Merge of EGFP immunostaining and DAPI. Scale bar: $100 \mu \mathrm{m}$. $\boldsymbol{B}$, qRT-PCR analysis of Tet $7^{F L}$ expression levels in the dHPC two weeks after AAV1-Tet7 $7^{F L}$-NFD, - Tet7 ${ }^{F L}$-SID4X and -Tet7 ${ }^{S}$-SID4X transduction; $* p<0.05$ (Dunnett's post hoc test); $n=8$. C, qRT-PCR analysis of Tet $7^{S}$ expression levels in the dHPC

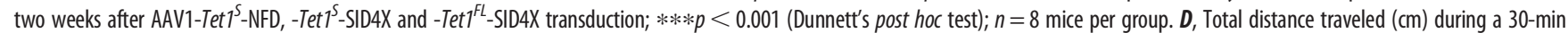
open field exploration test in AAV1-Tet7 ${ }^{F L}$-NFD and -Tet7 ${ }^{F L}$-SID4X transduced mice; $n=11-12$ mice per group. $\boldsymbol{E}$, Total distance traveled (cm) during a 30-min open field exploration test in AAV1-Tet $7^{S}$-NFD and -Tet $7^{S}$-SID4X transduced mice; $n=11-13$ mice per group. $\boldsymbol{F}$, Percent time in the open arms during 5 min of exploration in the EZM in AAV1-Tet $7^{F L}$-NFD and -Tet $7^{F L}$-SID4X transduced mice; $n=11-12$ mice per group. G, Percent time in the open arms during 5 min of exploration in the EZM in AAV1-Tet $1^{S}$-NFD and -Tet $T^{S}$-SID4X transduced mice; $n=11-13$ mice per group. $\boldsymbol{H}$, Percent time spent freezing in AAV1-Tet ${ }^{F L}$-NFD and -Tet7 ${ }^{F L}$-SID4X transduced mice during a 3.5-min CxFC training session; $n=11-12$ mice per group. $\boldsymbol{I}$, Percent time spent freezing in AAV1-Tet $7^{S}$-NFD and - Tet $7^{S}$-SID4X transduced mice during a 3.5-min CXFC training session; $n=11-13$ mice per group. J, Percent time spent freezing in AAV1-Tet $7^{F L}$-NFD and - Tet $7^{F L}$ SID4X transduced mice during a 5-min (XFC test $24 \mathrm{~h}$ after training; $* p<0.05$ (unpaired two-tailed $t$ test); $n=11-12$ mice per group. $K$, Percent time spent freezing in AAV1-Tet $7^{5}$-NFD and -Tet $T^{5}$-SID4X transduced mice during a 5-min CXFC test $24 \mathrm{~h}$ after training; $* * p<0.01$ (unpaired two-tailed $t$ test); $n=11-13$ mice per group. All data represent mean \pm SEM.

$3520 \pm 28, n=12$ vs Tet $1^{F L}-$ SID $4 X, 4322 \pm 35, n=11, t_{(21)}=$ $0.64, p=0.53$, and $T e t 1^{S}$-NFD, $3746 \pm 38, n=11$ vs Tet $1^{S}$-SID 4 X, $3459 \pm 26, n=13, t_{(20)}=1.8, p=0.0908$; unpaired two-tailed $t$ test: EZM, percent time in open: $\operatorname{Tet}^{F L}-\mathrm{NFD}, 47 \pm 2.5 \%, n=12$ vs Tet $1^{\text {FL }}$-SID $4 \mathrm{X}, 47 \pm 3 \%, n=11, t_{(21)}=0.0041, p=1$, and Tet ${ }^{S}$ NFD, $48 \pm 4.2 \%, n=11$ vs Tet $1^{S}$-SID $4 X, 57 \pm 2.8 \%, n=13$, $t_{(22)}=1.8, p=0.84$; unpaired two-tailed $t$ test], suggesting that manipulation of $\operatorname{Tet}^{F L}$ or Tet $1^{S}$ in the dHPC does not affect general locomotion (Fig. 7D,E) or basal anxiety levels (Fig. 7F,G).

Because previous studies examining the role of TET1 in memory formation were conducted in mice with disruptions that affect both isoforms (Rudenko et al., 2013; Zhang et al., 2013; Kumar et al., 2015; Towers et al., 2018), we next examined whether repression of either Tet $1^{F L}$ or $T e t 1^{S}$ alone was sufficient to alter hippocampal-dependent memory formation. To test this, we trained mice using a moderate (see Materials and Methods) CxFC paradigm and tested them $24 \mathrm{~h}$ later, with the percentage of time spent freezing serving as an indirect measure of associative memory formation. We found no differences in the percentage of time freezing during training between Tet $1^{F L}$ SID4X and Tet $1^{F L}$-NFD mice (percent time freezing: Tet ${ }^{F L}$ NFD, $8.1 \pm 2.8 \%, n=12$ vs Tet $1^{F L}$-SID $4 X, 3.6 \pm 1.3 \%, n=11$, $t_{(21)}=1.4, p=0.18$; unpaired two-tailed $t$ test), nor between the Tet $1^{S}$-SID4X and $T e t 1^{S}$-NFD groups (percent time freezing: Tet $1^{S}$-NFD, $3.6 \pm 1.2 \%, n=11$ vs Tet $1^{S}$-SID $4 X, 7 \pm 1.8 \%$, $n=13, t_{(22)}=1.5, p=0.14$; unpaired two-tailed $t$ test), suggesting depletion of Tet $1^{F L}$ or Tet ${ }^{S}$ in the dHPC does not affect baseline freezing levels (Fig. $7 \mathrm{H}, \mathrm{I}$ ). However, $24 \mathrm{~h}$ later, Tet ${ }^{F L}$ SID4X mice exhibited a reduction in their time spent freezing compared with Tet $^{F L}$-NFD controls (percent time freezing: Tet $1^{F L}$-NFD, $41 \pm 4.7 \%, n=12$ vs Tet ${ }^{F L}$-SID $4 X, 26 \pm 3.1 \%$, $n=11, t_{(21)}=2.6, p=0.017$; unpaired two-tailed $t$ test), suggesting impaired memory (Fig. $7 J)$. Moreover, Tet $1^{S}$-SID4X mice exhibited a significant increase in freezing time compared with Tet $1^{S}$-NFD controls (percent time freezing: Tet $1^{S}$-NFD, $38 \pm 2.6 \%, n=11$ vs Tet $^{S}$-SID $4 \mathrm{X}, 55 \pm 3.5 \%, n=13, t_{(22)}=3.8$, $p=0.0010$; unpaired two-tailed $t$ test), indicative of a memory enhancement (Fig. $7 \mathrm{~K}$ ). Together, these data demonstrate that hippocampal-dependent memory formation is bidirectionally modulated by the neuron-specific actions of each Tet 1 isoform.

\section{Discussion}

TET1 has been implicated in a wide variety of cognitive functions, most notably, learning and memory. However, the exact 
role of TET1 in the brain has remained ambiguous because of inconsistent findings reported between studies, suggesting key details regarding its function had yet to be elucidated. Here, we provide the first definitive evidence that two distinct isoforms of the Tet 1 gene are expressed in the adult mammalian brain. The first, Tet $1^{F L}$, is transcribed at low basal levels in neurons and encodes for the full-length canonical TET1 enzyme, while the second, $T e t 1^{S}$, is the predominately expressed isoform in the brain, enriched in neurons, and encodes for a recently discovered enzyme variant that lacks a large portion of the $\mathrm{N}$ terminus, including the CXXC DNA binding domain (Zhang et al., 2016). Using isoform-specific genetic tools, we find that the individual disruption of Tet $1^{F L}$ and $T e t 1^{S}$ in neurons has distinct effects on gene expression, excitatory synaptic transmission and memory formation, demonstrating that each isoform serves a non-redundant function in the nervous system.

In the first study to describe $T e t 1^{S}$, it was reported to be the only Tet 1 transcript expressed past early developmental stages (Zhang et al., 2016). However, others have shown in some adult tissues and/or cell types that both Tet1 isoforms are co-expressed (Good et al., 2017; Yosefzon et al., 2017). We found that while Tet $1^{S}$ is the predominantly expressed isoform in the brain, $\operatorname{Tet}^{F L}$ is also actively transcribed. We attribute these inconsistencies to differences in the experimental techniques used to detect the lowly expressed $T e t 1^{F L}$ transcript. For example, in the original report, a lack of $\mathrm{Tet}^{\mathrm{FL}}$ expression was inferred using genome-wide RNA-seq datasets, while more recent studies, including our own, have used more sensitive methods such as cell-type specific ChIP-seq, RNAP2 ChIP-qPCR, 5' RACE, and qRT-PCR.

We reported previously using immunohistochemistry that TET1 strongly co-localizes with the neuronal marker NeuN in the hippocampus, but only weakly with the astrocytic marker fibrillary acidic protein (GFAP), suggesting its enriched in neurons (Kaas et al., 2013). Our examination of Tet 1 isoform expression in neurons and glia supports these initial findings. For instance, while $\operatorname{Tet}^{S}$ is the most abundant transcript in both cell types, in relative terms, the short isoform is expressed at considerably higher levels in neurons $\left(\sim 3\right.$-fold), while in glia, Tet $1^{F L}$ was $\sim 15$-fold more abundant. Thus, the higher TET1 enrichment in neurons stems from greater $T e t 1^{S}$ expression, whereas in glia, despite significantly higher levels of $T e t 1^{F L}$, exhibits lower overall expression because of reduced levels of the short transcript. These findings also suggest that the role of each isoform may be at least partially cell-type specific. In support of this idea, Tet $1^{S}$ transcript levels are downregulated in cultured neurons following depolarization and after fear learning in hippocampal CA1, whereas $T e t 1^{F L}$, which is much less abundant in excitable cells, remained at baseline levels. Similarly, greater relative expression of $\operatorname{Tet}^{F L}$ in glia may reflect its reported role as a tumor suppressor in gliomas (Fu et al., 2017), where its added presence might be necessary to control gene expression programs related to cellular proliferation.

Several studies using pan-Tet1 $\mathrm{KO}$ mice have found that the loss of both isoforms provides cognitive benefits, including memory enhancement (Rudenko et al., 2013; Kumar et al., 2015; Feng et al., 2017; Cheng et al., 2018). Our findings that Tet $1^{S}$ repression alone is sufficient to enhance long-term fear memory suggests these pro-cognitive effects result from loss of the short isoform. These data are also consistent with our previous finding that acute overexpression of the TET1 catalytic domain, which also lacks the N-terminal domain of the full length enzyme, impairs memory formation (Kaas et al., 2013). Together, these observations strongly point to $\operatorname{Tet}^{S}$ as a memory suppressor, and perhaps more generally, as a negative regulator of neuroplasticity. In contrast, Tet ${ }^{F L}$ repression causes memory deficits. In agreement with these results, a recent study found that transgenic overexpression of Tet $1^{F L}$ causes enhanced memory formation and increased anxiety in mice (Kwon et al., 2018), indicating that overexpression of the full-length enzyme has the opposite effect on cognition. It is important to note that while our isoform-specific behavioral findings generally agree with previous reports, several groups have found that pan-Tet1 deficient mice to either have normal memory or even exhibit an impairment (Zhang et al., 2013; Towers et al., 2018). The cause of these conflicting findings is still not clear. It seems likely, as previously posited (for review, see Alaghband et al., 2016; Antunes et al., 2019), that these differences reflect the use of different KO strategies in mice that target different exons and/or the presence of developmental confounds; the latter being particularly relevant as some Tet1 mutant alleles display embryonic semilethality and/or smaller stature than littermates (Dawlaty et al., 2011; Kang et al., 2015; Towers et al., 2018).

Consistent with our behavioral data, individual disruption of Tet $1^{F L}$ and $T e t 1^{S}$ in hippocampal neuron cultures had dissimilar effects on excitatory synaptic transmission. In particular, Tet $1^{S}$ depletion led to a significant reduction in mEPSC frequency. This presynaptically driven process has been shown to inversely correlate with the strength of long-term depression (LTD) in hippocampal slices (Zhang et al., 2005). Indeed, Rudenko et al. (2013) reported enhanced LTD in hippocampal slices from panTet1 KO mice with heightened memory retention. LTD has been shown to be necessary and sufficient to facilitate long-term spatial memory formation (Ge et al., 2010; Dong et al., 2012b), thus providing a rationale for how reduced mEPSC frequency might lead to enhanced memory in $T e t 1^{S}$-deficient mice. In contrast, transcriptional repression of Tet $1^{F L}$ caused both an increase in mEPSC frequency and amplitude. While more work is required to resolve why this is, our behavioral findings suggest that Tet $1^{F L}$ normally acts to suppress aberrant hyperexcitability that can lead to cognitive impairment.

Our transcriptomic data provides important insights into how $T e t 1^{F L}$ and $T e t 1^{S}$ repression alters neuronal physiology and cognition. For instance, loss of Tet $1^{F L}$ disrupted the expression of a large swath of the neuronal genome, causing a significant upregulation of cancer and immune response pathways as well as the downregulation of genes important for neuronal physiology and learning. These data point to the canonical isoform as a critical regulator of genomic stability in neurons and provides a straightforward explanation for the impaired memory in Tet $1^{F L}$ deficient mice. Similarly, the hyperexcitability in Tet $1^{F L}$-depleted neurons likely results, in part, from the induction of immune response genes. In particular, those associated with the tumor necrosis factor (Tnf) pathway, as its activation via the cytokine TNF $\alpha$ has been shown to be sufficient to increase excitability (Ming et al., 2013). Interestingly, previous transcriptomic analyses of constitutive pan-Tet1 KO mice have not reported this activation of immune response genes (Rudenko et al., 2013; Zhang et al., 2013; Towers et al., 2018). We propose that the absence of an inflammatory response in these studies involves compensatory mechanisms during development, as viral-mediated conditional pan-Tet1 $\mathrm{KO}$ in the nucleus accumbens has been shown to strongly induce immune gene expression (Feng et al., 2017). In the case of $\operatorname{Tet}^{S}$, our transcriptomic data suggests that repression of the novel isoform may improve memory, by enhancing translation in neurons, as its loss resulted in the significant upregulation of genes encoding ribosomal RNAs (rRNAs) and 
proteins involved in ribosomal biogenesis. Consistent with this idea, a recent study has shown that learning-induced changes in rRNA expression are required for memory consolidation (Allen et al., 2018).

Although we do not address the molecular functions of the $\mathrm{TET} 1^{\mathrm{FL}}$ and $\mathrm{TET} 1^{\mathrm{S}}$ proteins in our study, recent findings provide some insights. For instance, the CXXC DNA binding domaindeficient TET $1^{\mathrm{S}}$ enzyme has been shown to exhibit lower chromatin affinity than $\mathrm{TET} 1^{\mathrm{FL}}$ when exogenously expressed in mESCs, yet still localizes to many of the same genomic features and gene targets as the canonical protein (Zhang et al., 2016). In addition, TET ${ }^{\mathrm{S}}$ expression resulted in a smaller increase in $5 \mathrm{hmC}$ than $\mathrm{TET1}^{\mathrm{FL}}$, suggesting it might be a less potent, or more selective, methylcytosine dioxygenase. Both findings provide a rationale for the overlap we observed in DEGs between isoforms, and why $\operatorname{Tet}^{S}$ repression resulted in milder changes in gene expression than Tet $1^{F L}$. Given that the CXXC domain directs $\mathrm{TET}^{\mathrm{FL}}$ to promoter-associated CpG islands (Xu et al., 2011), how $\mathrm{TET}^{\mathrm{S}}$ is recruited to its genomic targets remains an open question, but likely includes interactions with yet-to-be identified co-regulatory proteins. Recent published data suggests it might involve the transcription factors EGR1 (Sun et al., 2019) and FOXA1 (Yang et al., 2016), as blotting with a TET1 antibody after co-immunoprecipitation of these factors detected a band around $150 \mathrm{kDa}$, consistent with the predicted size of TET1 ${ }^{\mathrm{S}}$. Nevertheless, future studies addressing the molecular functions of Tet $1^{S}$ and $\operatorname{Tet} 1^{F L}$ will be needed to fully understand their roles in regulating nervous system function.

In conclusion, $\operatorname{Tet}^{F L}$ and $T e t 1^{S}$ are co-expressed in the adult brain, and carry out distinct functions, providing important new insights into the role of TET enzymes in the nervous system. Tet ${ }^{S}$ repression enhances memory formation, suggesting that antagonists selective for the truncated enzyme may be an effective therapeutic strategy to treat cognitive deficits. Tet $1^{F L}$, on the other hand, appears to be a critical regulator of neuroinflammation and cellular identity, suggestive of a role in aging, neurodegeneration and cancer. Overall, our results stress the importance of distinguishing between the two isoforms in future studies and provide the impetus to reexamine previous findings related to TET1 in depression, addiction and bipolar disorder (Dong et al., 2012a; Feng et al., 2015, 2017).

\section{References}

Alaghband Y, Bredy TW, Wood MA (2016) The role of active DNA demethylation and Tet enzyme function in memory formation and cocaine action. Neurosci Lett 625:40-46.

Allen KD, Regier MJ, Hsieh C, Tsokas P, Barnard M, Phatarpekar S, Wolk J, Sacktor TC, Fenton AA, Iván Hernández A (2018) Learning-induced ribosomal RNA is required for memory consolidation in mice-Evidence of differentially ex-pressed rRNA variants in learning and memory. PLoS One 13:e0203374.

Antunes C, Sousa N, Pinto L, Marques CJ (2019) TET enzymes in neurophysiology and brain function. Neurosci Biobehav Rev 102:337344.

Antunes C, Da Silva JD, Guerra-Gomes S, Alves ND, Ferreira F, LoureiroCampos E, Branco MR, Sousa N, Reik W, Pinto L, Marques CJ (2020) Tet3 ablation in adult brain neurons increases anxiety-like behavior and regulates cognitive function in mice. Mol Psychiatry. Advance online publication. Retrieved February 26, 2020. doi:10.1038/s41380-020-0695-7.

Beck DB, Petracovici A, He C, Moore HW, Louie RJ, Ansar M, Douzgou S, Sithambaram S, Cottrell T, Santos-Cortez RLP, Prijoles EJ, Bend R, Keren B, Mignot C, Nougues MC, Ounap K, Reimand T, Pajusalu S, Zahid M, Saqib MAN, et al. (2020) Delineation of a human Mendelian disorder of the DNA demethylation machinery: TET3 deficiency. Am J Hum Genet 106:234-245.

Bernstein BE, Mikkelsen TS, Xie X, Kamal M, Huebert DJ, Cuff J, Fry B, Meissner A, Wernig M, Plath K, Jaenisch R, Wagschal A, Feil R, Schreiber SL, Lander ES (2006) A bivalent chromatin structure marks key developmental genes in embryonic stem cells. Cell 125:315-326.

Bolger AM, Lohse M, Usadel B (2014) Trimmomatic: a flexible trimmer for Illumina sequence data. Bioinformatics 30:2114-2120.

Cheng Y, Sun M, Chen L, Li Y, Lin L, Yao B, Li Z, Wang Z, Chen J, Miao Z, Xin N, Huang L, Allen EG, Wu H, Xu X, Jin P (2018) Ten-eleven translocation proteins modulate the response to environmental stress in mice. Cell Rep 25:3194-3203.e4.

Choi JH, Yu NK, Baek GC, Bakes J, Seo D, Nam HJ, Baek SH, Lim CS, Lee YS, Kaang BK (2014) Optimization of AAV expression cassettes to improve packaging capacity and transgene expression in neurons. Mol Brain 7:17.

Cochran JN, Geier EG, Bonham LW, Newberry JS, Amaral MD, Thompson ML, Lasseigne BN, Karydas AM, Roberson ED, Cooper GM, Rabinovici GD, Miller BL, Myers RM, Yokoyama JS (2020) Non-coding and Lossof-Function Coding Variants in TET2 are Associated with Multiple Neurodegenerative Diseases. Am J Hum Genet 106:632-645.

Collins BE, Sweatt JD, Greer CB (2019) Broad domains of histone 3 lysine 4 trimethylation are associated with transcriptional activation in CA1 neurons of the hippocampus during memory formation. Neurobiol Learn Mem 161:149-167.

Dawlaty MM, Ganz K, Powell BE, Hu YC, Markoulaki S, Cheng AW, Gao Q, Kim J, Choi SW, Page DC, Jaenisch R (2011) Tet1 is dispensable for maintaining pluripotency and its loss is compatible with embryonic and postnatal development. Cell Stem Cell 9:166-175.

Dong E, Gavin DP, Chen Y, Davis J (2012a) Upregulation of TET1 and downregulation of APOBEC3A and APOBEC3C in the parietal cortex of psychotic patients. Transl Psychiatry 2:e159.

Dong Z, Gong B, Li H, Bai Y, Wu X, Huang Y, He W, Li T, Wang YT (2012b) Mechanisms of hippocampal long-term depression are required for memory enhancement by novelty exploration. J Neurosci 32:1198011990.

Dong E, Ruzicka WB, Grayson DR, Guidotti A (2015) DNA-methyltransferase1 (DNMT1) binding to $\mathrm{CpG}$ rich GABAergic and BDNF promoters is increased in the brain of schizophrenia and bipolar disorder patients. Schizophr Res 167:35-41.

Doyle EL, Booher NJ, Standage DS, Voytas DF, Brendel VP, Vandyk JK, Bogdanove AJ (2012) TAL effector-nucleotide targeter (TALE-NT) 2.0: tools for TAL effector design and target prediction. Nucleic Acids Res 40: W117-W122.

Feng J, Shao N, Szulwach KE, Vialou V, Huynh J, Zhong C, Le T, Ferguson D, Cahill ME, Li Y, Koo JW, Ribeiro E, Labonte B, Laitman BM, Estey D, Stockman V, Kennedy P, Couroussé T, Mensah I, Turecki G, et al. (2015) Role of Tet1 and 5-hydroxymethylcytosine in cocaine action. Nat Neurosci 18:536-544.

Feng J, Pena CJ, Purushothaman I, Engmann O, Walker D, Brown AN, Issler O, Doyle M, Harrigan E, Mouzon E, Vialou V, Shen L, Dawlaty MM, Jaenisch R, Nestler EJ (2017) Tet1 in nucleus accumbens opposes depression- and anxiety-like behaviors. Neuropsychopharmacology 42:16571669.

Fu R, Ding Y, Luo J, Yu L, Li CL, Li DS, Guo SW (2017) TET1 exerts its tumour suppressor function by regulating autophagy in glioma cells. Biosci Rep 37:BSR20160523.

Gates LA, Shi J, Rohira AD, Feng Q, Zhu B, Bedford MT, Sagum CA, Jung SY, Qin J, Tsai MJ, Tsai SY, Li W, Foulds CE, O’Malley BW (2017) Acetylation on histone $\mathrm{H} 3$ lysine 9 mediates a switch from transcription initiation to elongation. J Biol Chem 292:14456-14472.

Ge Y, Dong Z, Bagot RC, Howland JG, Phillips AG, Wong TP, Wang YT (2010) Hippocampal long-term depression is required for the consolidation of spatial memory. Proc Natl Acad Sci USA 107:1669716702.

Giardina C, Lis JT (1993) DNA melting on yeast RNA polymerase II promoters. Science 261:759-762.

Gibson DG, Young L, Chuang R-Y, Venter JC, Hutchison CA, Smith HO (2009) Enzymatic assembly of DNA molecules up to several hundred kilobases. Nat Methods 6:343-345. 
Good CR, Madzo J, Patel B, Maegawa S, Engel N, Jelinek J, Issa JPJ (2017) A novel isoform of TET1 that lacks a CXXC domain is overexpressed in cancer. Nucleic Acids Res 45:8269-8281.

Guo JU, Su Y, Zhong C, Ming GL, Song H (2011) Hydroxylation of 5-methylcytosine by TET1 promotes active DNA demethylation in the adult brain. Cell 145:423-434.

Halder R, Hennion M, Vidal RO, Shomroni O, Rahman RU, Rajput A, Centeno TP, Van Bebber F, Capece V, Vizcaino JCG, Schuetz AL, Burkhardt S, Benito E, Sala MN, Javan SB, Haass C, Schmid B, Fischer A, Bonn S (2016) DNA methylation changes in plasticity genes accompany the formation and maintenance of memory. Nat Neurosci 19:102-110.

Heinz S, Benner C, Spann N, Bertolino E, Lin YC, Laslo P, Cheng JX, Murre C, Singh H, Glass CK (2010) Simple combinations of lineage-determining transcription factors prime cis-regulatory elements required for macrophage and B cell identities. Mol Cell 38:576-589.

Hermann A, Goyal R, Jeltsch A (2004) The Dnmt1 DNA-(cytosineC5)-methyltransferase methylates DNA processively with high preference for hemimethylated target sites. J Biol Chem 279:4835048359.

Huang S, Wang Z, Zhou J, Huang J, Zhou L, Luo J, Wan YY, Long H, Zhu B (2019) EZH2 inhibitor GSK126 suppresses antitumor immunity by driving production of myeloid-derived suppressor cells. Cancer Res 79:20092020.

Hui CW, Zhang Y, Herrup K (2016) Non-neuronal cells are required to mediate the effects of neuroinflammation: results from a neuronenriched culture system. PLoS One 11:e0147134.

Jarome TJ Lubin FD (2014) Epigenetic mechanisms of memory formation and reconsolidation. Neurobiol Learn Mem 115:116-127.

Juillerat A, Dubois G, Valton J, Thomas S, Stella S, Maréchal A, Langevin S, Benomari N, Bertonat C, Silva GH, Daboussi F, Epinat JC, Montoya G, Duclert A, Duchateau P (2014) Comprehensive analysis of the specificity of transcription activator-like effector nucleases. Nucleic Acids Res 42:5390-5402.

Kaas G, Zhong C, Eason D, Ross D, Vachhani R, Ming G-L, King J, Song H, Sweatt J (2013) TET1 controls CNS 5-methylcytosine hydroxylation, active DNA demethylation, gene transcription, and memory formation. Neuron 79:1086-1093.

Kang J, Lienhard M, Pastor WA, Chawla A, Novotny M, Tsagaratou A, Lasken RS, Thompson EC, Azim Surani M, Koralov SB, Kalantry S, Chavez L, Rao A (2015) Simultaneous deletion of the methylcytosine oxidases Tet 1 and Tet 3 increases transcriptome variability in early embryogenesis. Proc Natl Acad Sci USA 112:E4236-E4245.

Kim D, Langmead B, Salzberg SL (2015) HISAT: a fast spliced aligner with low memory requirements. Nat Methods 12:357-360.

Konermann S, Brigham MD, Trevino AE, Hsu PD, Heidenreich M, Cong L, Platt RJ, Scott DA, Church GM, Zhang F (2013) Optical control of mammalian endogenous transcription and epigenetic states. Nature 500:472476.

Kügler S, Kilic E, Bähr M (2003) Human synapsin 1 gene promoter confers highly neuron-specific long-term transgene expression from an adenoviral vector in the adult rat brain depending on the transduced area. Gene Ther 10:337-347.

Kumar D, Aggarwal M, Kaas GA, Lewis J, Wang J, Ross DL, Zhong C, Kennedy A, Song H, Sweatt JD (2015) Tet1 oxidase regulates neuronal gene transcription, active DNA hydroxymethylation, object location memory, and threat recognition memory. Neuroepigenetics 4:12-27.

Kwon W, Kim HS, Jeong J, Sung Y, Choi M, Park S, Lee J, Jang S, Kim SH, Lee S, Kim MO, Ryoo ZY (2018) Tet1 overexpression leads to anxiety-like behavior and enhanced fear memories via the activation of calcium-dependent cascade through Egr1 expression in mice. FASEB J 32:390-403.

Lachmann A, Xu H, Krishnan J, Berger SI, Mazloom AR, Ma'ayan A (2010) ChEA: transcription factor regulation inferred from integrating genomewide ChIP-X experiments. Bioinformatics 26:2438-2444.

Langmead B, Trapnell C, Pop M, Salzberg SL (2009) Ultrafast and memoryefficient alignment of short DNA sequences to the human genome. Genome Biol 10:R25.

Leenen FAD, Vernocchi S, Hunewald OE, Schmitz S, Molitor AM, Muller CP, Turner JD (2016) Where does transcription start? 5'RACE adapted to next-generation sequencing. Nucleic Acids Res 44:2628-2645.
Li H, Handsaker B, Wysoker A, Fennell T, Ruan J, Homer N, Marth G, Abecasis G, Durbin R; 1000 Genome Project Data Processing Subgroup (2009) The sequence alignment/map format and SAMtools. Bioinformatics 25:2078-2079.

Liang G, Lin JCY, Wei V, Yoo C, Cheng JC, Nguyen CT, Weisenberger DJ, Egger G, Takai D, Gonzales FA, Jones PA (2004) Distinct localization of histone $\mathrm{H} 3$ acetylation and $\mathrm{H} 3-\mathrm{K} 4$ methylation to the transcription start sites in the human genome. Proc Natl Acad Sci USA 101:7357-7462.

Liao Y, Smyth GK, Shi W (2014) FeatureCounts: an efficient general purpose program for assigning sequence reads to genomic features. Bioinformatics 30:923-930.

Livak KJ, Schmittgen TD (2001) Analysis of relative gene expression data using real-time quantitative PCR and the $2-\Delta \Delta \mathrm{CT}$ method. Methods 25:402-408.

Martinowich K, Hattori D, Wu H, Fouse S, He F, Hu Y, Fan G, Sun YE (2003) DNA methylation-related chromatin remodeling in activity-dependent Bdnf gene regulation. Science 302:890-893.

McCarthy DJ, Chen Y, Smyth GK (2012) Differential expression analysis of multifactor RNA-Seq experiments with respect to biological variation. Nucleic Acids Res 40:4288-4297.

Mendenhall EM, Williamson KE, Reyon D, Zou JY, Ram O, Joung JK, Bernstein BE (2013) Locus-specific editing of histone modifications at endogenous enhancers. Nat Biotechnol 31:1133-1136.

Miller CA, Sweatt JD (2007) Covalent modification of DNA regulates memory formation. Neuron 53:857-869.

Ming Z, Criswell HE, Breese GR (2013) Evidence for TNF $\alpha$ action on excitatory and inhibitory neurotransmission in the central amygdala: a brain site influenced by stress. Brain Behav Immun 33:102-111.

Okano M, Bell DW, Haber DA, Li E (1999) DNA methyltransferases Dnmt3a and Dnmt3b are essential for de novo methylation and mammalian development. Cell 99:247-257.

Pertea M, Pertea GM, Antonescu CM, Chang TC, Mendell JT, Salzberg SL (2015) StringTie enables improved reconstruction of a transcriptome from RNA-seq reads. Nat Biotechnol 33:290-295.

Polstein LR, Perez-Pinera P, Kocak DD, Vockley CM, Bledsoe P, Song L, Safi A, Crawford GE, Reddy TE, Gersbach CA (2015) Genome-wide specificity of DNA binding, gene regulation, and chromatin remodeling by TALE- and CRISPR/Cas9-based transcriptional activators. Genome Res 25:1158-1169.

Robinson MD, McCarthy DJ, Smyth GK (2010) edgeR: a bioconductor package for differential expression analysis of digital gene expression data. Bioinformatics 26:139-140.

Rudenko A, Dawlaty MM, Seo J, Cheng AW, Meng J, Le T, Faull KF, Jaenisch R, Tsai LH (2013) Tet1 is critical for neuronal activity-regulated gene expression and memory extinction. Neuron 79:1109-1122.

Sanjana NE, Cong L, Zhou Y, Cunniff MM, Feng G, Zhang F (2012) A transcription activator-like effector toolbox for genome engineering. Nat Protoc 7:171-192

Sato Y, Hilbert L, Oda H, Wan Y, Heddleston JM, Chew TL, Zaburdaev V, Keller P, Lionnet T, Vastenhouw N, Kimura H (2019) Histone H3K27 acetylation precedes active transcription during zebrafish zygotic genome activation as revealed by live-cell analysis. Development 146: dev179127.

Saunderson EA, Spiers H, Mifsud KR, Gutierrez-Mecinas M, Trollope AF, Shaikh A, Mill J, Reul JMHM (2016) Stress-induced gene expression and behavior are controlled by DNA methylation and methyl donor availability in the dentate gyrus. Proc Natl Acad Sci USA 113:4830-4835.

Sun Z, Xu X, He J, Murray A, Sun M-a, Wei X, Wang X, McCoig E, Xie E, Jiang X, Li L, Zhu J, Chen J, Morozov A, Pickrell AM, Theus MH, Xie H (2019) EGR1 recruits TET1 to shape the brain methylome during development and upon neuronal activity. Nat Commun 10:3892.

Tahiliani M, Koh KP, Shen Y, Pastor WA, Bandukwala H, Brudno Y, Agarwal S, Iyer LM, Liu DR, Aravind L, Rao A (2009) Conversion of 5methylcytosine to 5-hydroxymethylcytosine in mammalian DNA by MLL partner TET1. Science 324:930-935.

Towers AJ, Tremblay MW, Chung L, Li X, Bey AL, Zhang W, Cao X, Wang X, Wang P, Duffney LJ, Siecinski SK, Xu S, Kim Y, Kong X, Gregory S, Xie W, Jiang Y (2018) Epigenetic dysregulation of Oxtr in Tet1-deficient mice has implications for neuropsychiatric disorders. JCI Insight 3: e120592. 
Verma SK, Tian X, LaFrance LV, Duquenne C, Suarez DP, Newlander KA, Romeril SP, Burgess JL, Grant SW, Brackley JA, Graves AP, Scherzer DA, Shu A, Thompson C, Ott HM, Aller GS, Machutta CA, Diaz E, Jiang Y, Johnson NW, et al. (2012) Identification of potent, selective, cell-Active inhibitors of the histone lysine methyltransferase EZH2. ACS Med Chem Lett 3:1091-1096.

Widagdo J, Li X, Wei W, D’Alessio A, Bredy TW, Zhao QY, Baker-Andresen D, Flavell CR, Zhang Y (2014) Neocortical Tet3-mediated accumulation of 5-hydroxymethylcytosine promotes rapid behavioral adaptation. Proc Natl Acad Sci USA 111:7120-7125.

Xu Y, Wu F, Tan L, Kong L, Xiong L, Deng J, Barbera AJ, Zheng L, Zhang H, Huang S, Min J, Nicholson T, Chen T, Xu G, Shi Y, Zhang K, Shi YG (2011) Genome-wide regulation of $5 \mathrm{hmC}, 5 \mathrm{mC}$, and gene expression by Tet1 hydroxylase in mouse embryonic stem cells. Mol Cell 42:451-464

Yang YA, Zhao JC, Fong KW, Kim J, Li S, Song C, Song B, Zheng B, He C, Yu J (2016) FOXA1 potentiates lineage-specific enhancer activation through modulating TET1 expression and function. Nucleic Acids Res 44:8153-8164.

Yosefzon Y, David C, Tsukerman A, Pnueli L, Qiao S, Boehm U, Melamed P (2017) An epigenetic switch repressing Tetl in gonadotropes activates the reproductive axis. Proc Natl Acad Sci USA 114:10131-10136.
Yu H, Su Y, Shin J, Zhong C, Guo JU, Weng YL, Gao F, Geschwind DH, Coppola G, Ming GL, Song H (2015) Tet3 regulates synaptic transmission and homeostatic plasticity via DNA oxidation and repair. Nat Neurosci 18:836-843.

Zengeler KE, Gettens CP, Smith HC, Caron MM, Zhang X, Howard AH, Boitnott AR, Gogliettino AR, Reda A, Malachowsky BG, Zhong C, Song H, Kaas GA, Kennedy AJ (2019) Tet2 negatively regulates memory fidelity. bioRxiv 843581 .

Zhang J, Yang Y, Li H, Cao J, Xu L (2005) Amplitude/frequency of spontaneous mEPSC correlates to the degree of long-term depression in the CA1 region of the hippocampal slice. Brain Res 1050:110-117.

Zhang RR, Cui QY, Murai K, Lim YC, Smith ZD, Jin S, Ye P, Rosa L, Lee YK, Wu HP, Liu W, Xu ZM, Yang L, Ding YQ, Tang F, Meissner A, Ding C, Shi Y, Xu GL (2013) Tet1 regulates adult hippocampal neurogenesis and cognition. Cell Stem Cell 13:237-245.

Zhang W, Xia W, Wang Q, Towers AJ, Chen J, Gao R, Zhang Y, Yen CA, Lee AY, Li Y, Zhou C, Liu K, Zhang J, Gu TP, Chen X, Chang Z, Leung D, Gao S, Jiang YH, Xie W (2016) Isoform switch of TET1 regulates DNA demethylation and mouse development. Mol Cell 64:1062-1073 